\title{
НЕЛИНЕЙНАЯ ЗАДАЧА ДЛЯ ПАРАБОЛИЧЕСКОГО УРАВНЕНИЯ С НЕИЗВЕСТНЫМ КОЭФФИЦИЕНТОМ ПРИ ПРОИЗВОДНОЙ ПО ВРЕМЕНИ И ЕЕ ПРИЛОЖЕНИЯ В МАТЕМАТИЧЕСКИХ МОДЕЛЯХ ФИЗИКО-ХИМИЧЕСКИХ ПРОЦЕССОВ
}

\begin{abstract}
Н. Л. Гольдман ${ }^{1}$
Рассматривается нелинейная система с неизвестным коэффициентом при производной по времени в параболическом уравнении и изучаются вопросы существования и единственности ее решения в классе гладких функций. В качестве способа доказательства разрешимости применяется метод прямых Ротэ, который является также и конструктивным методом приближенного решения. Для обоснования метода получены априорные оценки в сеточно-непрерывных классах Гельдера для соответствующей дифференциально-разностной нелинейной системы. Наличие таких оценок позволяет установить сходимость приближенных решений к гладкому решению исходной параболической системы и оценить погрешность метода прямых. Проведенное исследование связано с математическим моделированием физико-химических процессов, в которых происходят изменения внутренних характеристик материалов. Представлен пример задачи о деструкции теплозащитного композиционного материала при высокотемпературном нагреве.
\end{abstract}

Ключевые слова: параболические уравнения, классы Гельдера, метод прямых Ротэ, априорные оценки, однозначная разрешимость, математическая модель термодеструкции, композиционный материал.

1. Введение. Целый ряд современных технологий характеризуется физико-химическими процессами, в которых происходят изменения внутренних свойств материалов. В настоящей статье изучается нелинейная параболическая система, возникающая при применении математического моделирования к таким процессам. Изучение связано не только с практическим интересом к ее разнообразным приложениям. В силу сложности данной параболической системы значительный теоретический интерес представляет обоснование ее математической постановки. В связи с этим в статье рассматриваются условия существования и единственности решения в классе гладких функций, в том числе возможности применения метода прямых Ротэ для доказательства разрешимости исследуемой системы. Основу доказательства составляет получение априорных оценок в сеточно-непрерывных классах Гельдера для соответствующей дифференциально-разностной нелинейной системы, возникающей при применении метода прямых. Наличие таких оценок позволяет установить сходимость решений из сеточно-непрерывных классов Гельдера к гладкому решению исходной параболической системы и оценить погрешность метода прямых, что делает этот метод также и конструктивным способом приближенного решения.

Используемые в статье функциональные пространства определяются стандартным образом, как и в [1]. В частности, следуя [1], класс Гельдера $H^{2+\lambda, 1+\lambda / 2}(\bar{Q})(0<\lambda<1)$ определяется как пространство функций $u(x, t)$, непрерывных в замкнутой области $\bar{Q}=\{0 \leqslant x \leqslant l, 0 \leqslant t \leqslant T\}$ вместе со своими производными $u_{x x}, u_{t}$, которые удовлетворяют условию Гельдера по $x$ и $t$ с показателями $\lambda$ и $\lambda / 2$ соответственно.

Для удобства изложения будут использованы также следующие обозначения:

$H^{1, \lambda / 2,1}(\bar{D})$ - пространство функций, непрерывных при $(x, t, u) \in \bar{D}=\bar{Q} \times\left[-M_{0}, M_{0}\right]$, имеющих непрерывные в $\bar{D}$ производные по $x$ и $u$ и удовлетворяющих условию Гельдера по $t$ с показателем $\lambda / 2$;

$O^{1,0}(\bar{Q})$ - пространство непрерывных при $(x, t) \in \bar{Q}$ функций, имеющих ограниченные в $\bar{Q}$ производные по $x$;

$O^{1,0,1}(\bar{D})$ - пространство непрерывных при $(x, t, u) \in \bar{D}$ функций, имеющих ограниченные в $\bar{D}$ производные по $x$ и $u$;

$O^{2,0,2}(\bar{D})$ - пространство непрерывных при $(x, t, u) \in \bar{D}$ функций, имеющих непрерывные в $\bar{D}$ производные по $x$ и $u$ и ограниченные в $\bar{D}$ производные по $x x, x u$ и $u u$.

${ }^{1}$ Московский государственный университет им. М. В. Ломоносова, Научно-исследовательский вычислительный центр, Ленинские горы, 119992, Москва; вед. науч. сотр., e-mail: goldman@srcc.msu.ru

(c) Научно-исследовательский вычислительный центр МГУ им. М. В. Ломоносова 
Кроме того, используем аналоги классов Гельдера для сеточных функций $\hat{u}=\left(u_{0}, \ldots, u_{n}, \ldots, u_{N}\right)$, заданных в узлах $t_{n}$ сетки $\bar{\omega}_{\tau} \in[0, T]$ с шагом $\tau=T N^{-1}$, и для заданных в области $\bar{Q}_{\tau}=\left\{0 \leqslant x \leqslant l, t_{n} \in \bar{\omega}_{\tau}\right\}$ сеточно-непрерывных функций $\hat{u}(x)=\left(u_{0}(x), \ldots, u_{n}(x), \ldots, u_{N}(x)\right)$.

Как и в $[2,3]$, определим эти аналоги следующим образом.

$H_{\tau}^{1+\lambda / 2}\left(\bar{\omega}_{\tau}\right)$ - аналог пространства $H^{1+\lambda / 2}[0, T][1]$ для функций $\hat{u}$, имеющих конечную норму

$$
\begin{aligned}
|\hat{u}|_{\bar{\omega}_{\tau}}^{1+\lambda / 2} & =\max _{0 \leqslant n \leqslant N}\left|u_{n}\right|+\max _{1 \leqslant n \leqslant N}\left|u_{n \bar{t}}\right|+\left\langle\hat{u}_{\bar{t}}\right\rangle_{\bar{\omega}_{\tau}}^{\lambda / 2}, \\
u_{n \bar{t}} & =\left(u_{n}-u_{n-1}\right) \tau^{-1}, \quad n=\overline{1, N}, \\
\left\langle\hat{u}_{\bar{t}}\right\rangle_{\bar{\omega}_{\tau}}^{\lambda / 2} & =\max _{1 \leqslant n<n^{\prime} \leqslant N}\left\{\left|u_{n \bar{t}}-u_{n^{\prime} \bar{t}}\right|\left|t_{n}-t_{n^{\prime}}\right|^{-\lambda / 2}\right\} .
\end{aligned}
$$

$H_{\tau}^{\lambda, \lambda / 2}\left(\bar{Q}_{\tau}\right)$ - аналог пространства $H^{\lambda, \lambda / 2}(\bar{Q})[1]$ для функций $\hat{u}(x)$, непрерывных по $x$ при $\left(x, t_{n}\right) \in$ $\bar{Q}_{\tau}$ и обладающих конечной нормой

$$
\begin{aligned}
|\hat{u}(x)|_{\bar{Q}_{\tau}, \lambda / 2}^{\lambda, 2} & =\max _{\left(x, t_{n}\right) \in \bar{Q}_{\tau}}\left|u_{n}(x)\right|+\langle\hat{u}(x)\rangle_{x, \bar{Q}_{\tau}}^{\lambda}+\langle\hat{u}(x)\rangle_{t, \bar{Q}_{\tau}}^{\lambda / 2}, \\
\langle\hat{u}(x)\rangle_{x, \bar{Q}_{\tau}}^{\lambda} & =\sup _{\left(x, t_{n}\right),\left(x^{\prime}, t_{n}\right) \in \bar{Q}_{\tau}}\left\{\left|u_{n}(x)-u_{n}\left(x^{\prime}\right)\right|\left|x-x^{\prime}\right|^{-\lambda}\right\}, \\
\langle\hat{u}(x)\rangle_{t, \bar{Q}_{\tau}}^{\lambda / 2} & =\sup _{\left(x, t_{n}\right),\left(x, t_{n}^{\prime}\right) \in \bar{Q}_{\tau}}\left\{\left|u_{n}(x)-u_{n^{\prime}}(x)\right|\left|t_{n}-t_{n^{\prime}}\right|^{-\lambda / 2}\right\} .
\end{aligned}
$$

$H_{\tau}^{1+\lambda,(1+\lambda) / 2}\left(\bar{Q}_{\tau}\right)$ - аналог пространства $H^{1+\lambda,(1+\lambda) / 2}(\bar{Q})$ (см. [1]) для функций $\hat{u}(x)$, непрерывных по $x$ вместе со своими производными по $x$ при $\left(x, t_{n}\right) \in \bar{Q}_{\tau}$ и обладающих конечной нормой

$$
|\hat{u}(x)|_{\bar{Q}_{\tau}}^{1+\lambda,(1+\lambda) / 2}=\max _{\left(x, t_{n}\right) \in \bar{Q}_{\tau}}\left|u_{n}(x)\right|+\left|\hat{u}_{x}(x)\right|_{\bar{Q}_{\tau}}^{\lambda, \lambda / 2}+\langle\hat{u}(x)\rangle_{t, \bar{Q}_{\tau}}^{(1+\lambda) / 2} .
$$

$H_{\tau}^{2+\lambda, 1+\lambda / 2}\left(\bar{Q}_{\tau}\right)$ - аналог пространства $H^{2+\lambda, 1+\lambda / 2}(\bar{Q})$ для функций $\hat{u}(x)$, непрерывных по $x$ вместе со своими производными $\hat{u}_{x x}(x)$ и $\hat{u}_{t}(x)$ при $\left(x, t_{n}\right) \in \bar{Q}_{\tau}$ и обладающих конечной нормой

$$
\begin{gathered}
|\hat{u}(x)| \bar{Q}_{\tau}^{2+\lambda, 1+\lambda / 2}=\max _{\left(x, t_{n}\right) \in \bar{Q}_{\tau}}\left|u_{n}(x)\right|+\max _{\left(x, t_{n}\right) \in \bar{Q}_{\tau}}\left|u_{n x}(x)\right|+\left|\hat{u}_{x x}(x)\right| \bar{Q}_{\tau}^{\lambda, \lambda / 2}+\left|\hat{u}_{\bar{t}}(x)\right| \bar{Q}_{\tau}^{\lambda, \lambda / 2}, \quad \text { где } \\
\hat{u}_{x x}(x)=\left(u_{0 x x}(x), \ldots, u_{n x x}(x), \ldots, u_{N x x}(x)\right), \quad \hat{u}_{\bar{t}}(x)=\left(u_{1 \bar{t}}(x), \ldots, u_{n \bar{t}}(x), \ldots, u_{N \bar{t}}(x)\right), \\
u_{n \bar{t}}(x)=\left(u_{n}(x)-u_{n-1}(x)\right) \tau^{-1}, n=\overline{1, N} .
\end{gathered}
$$

2. Постановка нелинейной параболической системы с неизвестным коэффициентом при производной по времени. В области $\bar{Q}=\{0 \leqslant x \leqslant l, 0 \leqslant t \leqslant T\}$ требуется найти решение $\{u(x, t), \rho(x, t)\}$ системы, задаваемой условиями

$$
\begin{gathered}
c(x, t, u) \rho(x, t) u_{t}-\left(a(x, t, u) u_{x}\right)_{x}+b(x, t, u) u_{x}+d(x, t, u) u=f(x, t), \quad(x, t) \in Q, \\
a(x, t, u) u_{x}-\left.h(t, u) u\right|_{x=0}=g(t), \quad 0<t \leqslant T, \\
a(x, t, u) u_{x}+\left.e(t, u) u\right|_{x=l}=q(t), \quad 0<t \leqslant T, \\
\left.u\right|_{t=0}=\varphi(x), \quad 0 \leqslant x \leqslant l, \\
\rho_{t}(x, t)=\gamma(x, t, u), \quad(x, t) \in Q,\left.\quad \rho(x, t)\right|_{t=0}=\rho^{0}(x),
\end{gathered}
$$

в которых $a, b, c, d, f$, а также $h, e, g, q, \varphi, \gamma$ и $\rho^{0}$ - известные функции своих аргументов, $a \geqslant a_{\min }>0$, $c \geqslant c_{\min }>0, \rho^{0} \geqslant \rho_{\min }^{0}>0, h \geqslant 0, e \geqslant 0, a_{\min }, c_{\min }, \rho_{\min }^{0}=$ const $>0$.

В зависимости от знака функции $\gamma(x, t, u)$ при $(x, t, u) \in \bar{D}=\bar{Q} \times\left[-M_{0}, M_{0}\right]$ (где $M_{0} \geqslant \max _{(x, t) \in \bar{Q}}|u|, M_{0}-$ постоянная из принципа максимума для краевой задачи (1)-(4)) требование параболичности уравнения (1) приводит к ограничениям на искомое решение

$$
0<\rho_{\min }^{0}<\rho(x, t) \leqslant \max _{0 \leqslant x \leqslant l} \rho^{0}(x)+T \max _{(x, t, u) \in \bar{D}} \gamma(x, t, u) \quad \text { при } \quad \gamma(x, t, u)>0, \quad(x, t, u) \in \bar{D},
$$




$$
0<\rho_{\min }^{0}-T \max _{(x, t, u) \in \bar{D}}|\gamma(x, t, u)| \leqslant \rho(x, t) \leqslant \max _{0 \leqslant x \leqslant l} \rho^{0}(x) \quad \text { при } \quad \gamma(x, t, u) \leqslant 0, \quad(x, t, u) \in \bar{D} .
$$

Если $\gamma(x, t, u) \leqslant 0$ в области $\bar{D}$, то условие $(7)$ накладывает ограничение на отрезок времени $[0, T]$, на котором ищется решение системы (1)-(5): $0<T<\rho_{\min }^{0}\left(\max _{(x, t, u) \in \bar{D}}|\gamma(x, t, u)|\right)^{-1}$.

3. Обоснование метода прямых для системы (1)-(5). В качестве способа доказательства существования гладкого решения $\{u(x, t), \rho(x, t)\}$ системы (1)-(5) воспользуемся методом прямых Ротэ.

3.1. Нелинейная дифференциально-разностная система. Разбивая область $\bar{Q}$ на слои прямыми $t=t_{n}\left(t_{n}-\right.$ узлы равномерной сетки $\bar{\omega}_{\tau} \in[0, T]$ с шагом $\left.\tau=T N^{-1}\right)$, заменим систему $(1)-(5)$ дифференциально-разностной системой определения $\left\{u_{n}(x), \rho_{n}(x)\right\}$ - приближенных значений функций $u(x, t)$ и $\rho(x, t)$ при $t=t_{n}:$

$$
\begin{gathered}
c_{n} \rho_{n} u_{n \bar{t}}-\left(a_{n} u_{n x}\right)_{x}+b_{n} u_{n x}+d_{n} u_{n}=f_{n}, \quad\left(x, t_{n}\right) \in Q_{\tau}=\{0<x<l\} \times \omega_{\tau}, \\
a_{n} u_{n x}-\left.h_{n} u_{n}\right|_{x=0}=g_{n}, \quad 0<t_{n} \leqslant T, \\
a_{n} u_{n x}+\left.e_{n} u_{n}\right|_{x=l}=q_{n}, \quad 0<t_{n} \leqslant T, \\
u_{0}(x)=\varphi(x), \quad 0 \leqslant x \leqslant l, \\
\rho_{n \bar{t}}=\gamma_{n-1}, \quad\left(x, t_{n}\right) \in Q_{\tau},\left.\quad \rho_{n}\right|_{n=0}=\rho^{0}(x), \quad 0 \leqslant x \leqslant l,
\end{gathered}
$$

где $a_{n}, b_{n}, c_{n}, d_{n}$ - значения соответствующих коэффициентов в точке $\left(x, t_{n}, u_{n}\right), f_{n}=f\left(x, t_{n}\right), h_{n}$ и $e_{n}-$ значения коэффициентов в граничных условиях при $t=t_{n}, u=\left.u_{n}\right|_{x=0}$ и, соответственно, $u=\left.u_{n}\right|_{x=l}$,

$$
\begin{gathered}
g_{n}=g\left(t_{n}\right), \quad q_{n}=q\left(t_{n}\right), \quad \gamma_{n-1}=\gamma\left(x, t_{n-1}, u_{n-1}\right), \\
u_{n \bar{t}}=\left(u_{n}(x)-u_{n-1}(x)\right) \tau^{-1}, \quad u_{n x}=\frac{d u_{n}(x)}{d x}, \quad \rho_{n \bar{t}}=\left(\rho_{n}(x)-\rho_{n-1}(x)\right) \tau^{-1} .
\end{gathered}
$$

Разобьем доказательство разрешимости исходной задачи (1)-(5) методом прямых на несколько основных этапов.

Этап 1. Исследование дифференциально-разностной краевой задачи (8)-(11) в сеточно-непрерывном классе Гельдера $H_{\tau}^{2+\lambda, 1+\lambda / 2}\left(Q_{\tau}\right)$ в предположении, что $\rho_{n}(x)$ - известная функция. Получение соответствующих априорных оценок для $u_{n}(x)$, не зависящих от $x, \tau, n$. Доказательство существования и единственности решения $u_{n}(x) \in H_{\tau}^{2+\lambda, 1+\lambda / 2}\left(Q_{\tau}\right)$.

Этап 2. Получение априорных оценок в соответствующих сеточно-непрерывных классах для решения $\left\{u_{n}(x), \rho_{n}(x)\right\}$ всей дифференциально-разностной системы (8)-(12) на основе результатов этапа 1.

Этап 3. Предельный переход при $n \rightarrow \infty$ в условиях (8)-(12) на основе полученных априорных оценок компактности семейств $\left\{u_{n}(x), \rho_{n}(x)\right\}$. Завершение доказательства разрешимости нелинейной системы (1)-(5) в классе гладких функций.

Переходя к этим этапам, будем более подробно останавливаться лишь на тех моментах, которые связаны со спецификой задачи (1)-(5). При рассмотрении же моментов, которые являются общими для метода прямых при исследовании нелинейных параболических задач, ограничимся ссылками на известные результаты.

3.2. Дифференциально-разностная краевая задача (8)-(11). Предположим сначала, что $\rho_{n}(x)$ в уравнении $(8)$ - известная функция, ограниченная в области $\bar{Q}_{\tau}\left(0<\rho_{\min } \leqslant \rho_{n}(x) \leqslant \rho_{\max }, \rho_{\min }\right.$, $\rho_{\max }=$ const $\left.>0\right)$, имеющая непрерывную производную $\rho_{n x}(x)$ и удовлетворяющая условию Гельдера по $t$ с показателем $\lambda / 2$ при $\left(x, t_{n}\right) \in \bar{Q}_{\tau}$. Исходя из этого предположения, установим априорные оценки для решения $u_{n}(x)$ дифференциально-разностной краевой задачи (8)-(11).

\subsection{1. Оценка принципа максимума $\max _{\left(x, t_{n}\right) \in \bar{Q}_{\tau}}\left|u_{n}(x)\right|$.}

Лемма 1. Пусть при $(x, t) \in \bar{Q}$ и любых $u,|u|<\infty$, все входные данные задачи (1)-(4) являются ограниченными функииями, причем коэффициент $a(x, t, u)$ ограничен вместе со своими производными по $x$ u u. Предположим такэе, что $0<a_{\min } \leqslant a \leqslant a_{\max }, 0<c_{\min } \leqslant c \leqslant c_{\max }, 0<\rho_{\min } \leqslant \rho \leqslant \rho_{\max }$, $h \geqslant 0, e \geqslant 0$. Тогда в области $\bar{Q}_{\tau}$ при достаточно малых $\tau$ для любого решения $u_{n}(x)$ диффберенциальноразностной краевой задачи (8)-(11), аппроксимирующей задачу (1)-(4), имеет место оченка

$$
\max _{\left(x, t_{n}\right) \in \bar{Q}_{\tau}}\left|u_{n}(x)\right| \leqslant M_{0}, \quad M_{0}=K_{2} T \exp \left(K_{1} T\right)+K_{3} l\left(1+\frac{l}{4}\right),
$$


где $K_{1}, K_{2}, K_{3}=$ const $>0, \tau \leqslant \tau_{0}=\varepsilon K_{1}^{-1}, \varepsilon>0-$ любое, $K_{1} \geqslant(1+\varepsilon) d_{\max } c_{\min }^{-1} \rho_{\min }^{-1} u$

$$
\begin{aligned}
& K_{2} \geqslant c_{\min }^{-1} \rho_{\min }^{-1}\left\{f_{\max }+2 K_{3} a_{\max }+K_{3} l\left(a_{x \max }+K_{3} l a_{u \max }+b_{\max }+\left(1+\frac{l}{4}\right) d_{\max }\right)\right\}, \\
& K_{3} \geqslant \max \left(l^{-1} \varphi_{\max }, l^{-1} a_{\min }^{-1} g_{\max }, l^{-1} a_{\min }^{-1} q_{\max }\right) .
\end{aligned}
$$

Доказательство. Введем вспомогательную функцию $v_{n}(x)$, полагая

$$
u_{n}(x)=v_{n}(x)\left(1+K_{1} \tau\right)^{n}-\psi(x),
$$

где $K_{1}>0$ - некоторая постоянная, $\psi(x)$ - некоторая функция из $C^{2}[0, l]$, определим их позднее. Имеют место соотношения

$$
\begin{aligned}
u_{n \bar{t}} & =\tau^{-1}\left\{v_{n}\left(1+K_{1} \tau\right)^{n}-v_{n-1}\left(1+K_{1} \tau\right)^{n-1}\right\}= \\
& =\left(1+K_{1} \tau\right)^{n-1}\left\{\left(1+K_{1} \tau\right) v_{n \bar{t}}-K_{1} \tau v_{n \bar{t}}+K_{1} v_{n}\right\}=\left(1+K_{1} \tau\right)^{n-1} v_{n \bar{t}}+K_{1}\left(1+K_{1} \tau\right)^{n-1} v_{n}, \\
\left(a_{n} u_{n x}\right)_{x} & =\left(1+K_{1} \tau\right)^{n}\left(a_{n} v_{n x}\right)_{x}-2\left(1+K_{1} \tau\right)^{n} a_{n u} v_{n x} \psi_{x}-\left(a_{n} \psi_{x x}+a_{n x} \psi_{x}-a_{n u} \psi_{x}^{2}\right) .
\end{aligned}
$$

С учетом этих соотношений рассмотрим оператор $\mathcal{L} v_{n}$ следующего вида:

$$
\mathcal{L} v_{n} \equiv c_{n} \rho_{n} v_{n \bar{t}}-\left(1+K_{1} \tau\right)\left(a_{n} v_{n x}\right)_{x}+\left(1+K_{1} \tau\right)\left(b_{n}+2 a_{n u} \psi_{x}\right) v_{n x}+\left\{c_{n} \rho_{n} K_{1}+\left(1+K_{1} \tau\right) d_{n}\right\} v_{n} .
$$

Тогда в силу (8)-(11) функция $v_{n}(x)$ удовлетворяет следующей дифференциально-разностной краевой задаче:

$$
\begin{aligned}
\mathcal{L} v_{n} & =\left(1+K_{1} \tau\right)^{-(n-1)} F_{n}(x), \quad\left(x, t_{n}\right) \in Q_{\tau}, \\
a_{n} v_{n x}-\left.h_{n} v_{n}\right|_{x=0} & =\left.\left(1+K_{1} \tau\right)^{-n}\left(g_{n}+a_{n} \psi_{x}-h_{n} \psi\right)\right|_{x=0}, \quad 0<t_{n} \leqslant T, \\
a_{n} v_{n x}+\left.e_{n} v_{n}\right|_{x=l}= & \left.\left(1+K_{1} \tau\right)^{-n}\left(q_{n}+a_{n} \psi_{x}+e_{n} \psi\right)\right|_{x=l}, \quad 0<t_{n} \leqslant T, \\
& v_{0}(x)=\varphi(x)+\psi(x), \quad 0 \leqslant x \leqslant l,
\end{aligned}
$$

где $F_{n}(x)=f_{n}(x)-a_{n}(x) \psi_{x x}(x)-a_{n x}(x) \psi_{x}(x)+a_{n u}(x) \psi_{x}^{2}(x)+b_{n}(x) \psi_{x}(x)+d_{n}(x) \psi(x)$.

Далее, введем еще одну вспомогательную функцию $w_{n}(x)$ :

$$
w_{n}(x)=v_{n}(x)-K_{2} \tau n,
$$

где $K_{2}>0-$ постоянная, $K_{2} \geqslant c_{\min }^{-1} \rho_{\min }^{-1} \max _{\left(x, t_{n}\right) \in \bar{Q}_{\tau}}\left|F_{n}(x)\right|$. Как следствие уравнения (15), справедливо соотношение

$$
\mathcal{L} w_{n}=\left(1+K_{1} \tau\right)^{-(n-1)} F_{n}-c_{n} \rho_{n} K_{2}-\left\{c_{n} \rho_{n} K_{1}+\left(1+K_{1} \tau\right) d_{n}\right\} K_{2} \tau n .
$$

Выбираем теперь постоянную $K_{1}>0$ из условия $K_{1} \geqslant(1+\varepsilon) d_{\max } c_{\min }^{-1} \rho_{\min }^{-1}$, где $\varepsilon>0-$ любое. Это при достаточно малых $\tau, \tau \leqslant \tau_{0}=\varepsilon K_{1}^{-1}$, позволяет установить, что

$$
\mathcal{L} w_{n}(x)<0, \quad\left(x, t_{n}\right) \in Q_{\tau} .
$$

Кроме того, в силу (16)-(18) функция $w_{n}(x)$ удовлетворяет условиям

$$
\begin{gathered}
a_{n} w_{n x}-\left.h_{n} w_{n}\right|_{x=0}=\left.h_{n}\right|_{x=0} K_{2} \tau n+\left.\left(1+K_{1} \tau\right)^{-n}\left(g_{n}+a_{n} \psi_{x}-h_{n} \psi\right)\right|_{x=0}, \quad 0<t_{n} \leqslant T, \\
a_{n} w_{n x}+\left.e_{n} w_{n}\right|_{x=l}=-\left.e_{n}\right|_{x=l} K_{2} \tau n+\left.\left(1+K_{1} \tau\right)^{-n}\left(q_{n}+a_{n} \psi_{x}+e_{n} \psi\right)\right|_{x=l}, \quad 0<t_{n} \leqslant T, \\
w_{0}(x)=\varphi(x)+\psi(x), \quad 0 \leqslant x \leqslant l .
\end{gathered}
$$

Заметим, что во всех внутренних точках $\left(x, t_{n}\right) \in Q_{\tau}$ функция $w_{n}(x)$ неположительна. Действительно, иначе в точке положительного максимума $\left(x^{*}, t_{n^{*}}\right)$ выполнялись бы соотношения

$$
\left.w_{n^{*} \bar{t}}(x)\right|_{x=x^{*}} \geqslant 0,\left.\quad\left(a_{n^{*}}(x) w_{n^{*} x x}(x)\right)\right|_{x=x^{*}} \leqslant 0,\left.\quad w_{n^{*} x}(x)\right|_{x=x^{*}}=0,\left.\quad \mathcal{L} w_{n^{*}}(x)\right|_{x=x^{*}} \geqslant 0,
$$

что противоречит неравенству (20). Выберем теперь функцию $\psi(x)$ в $(14)$, полагая

$$
\psi(x)=-K_{3}\left(x-\frac{l}{2}\right)^{2}-K_{3} l
$$


где $K_{3}>0-$ постоянная, $K_{3} \geqslant \max \left(l^{-1} \varphi_{\max }, l^{-1} a_{\min }^{-1} g_{\max }, l^{-1} a_{\min }^{-1} q_{\max }\right)$.

При таком выборе $\psi(x)$ функция $w_{n}(x)$ неположительна и на границе области $\bar{Q}_{\tau}$. Действительно, если бы, например, при $x=\left.0 w_{n}(x)\right|_{x=0}>0$, то производная $\left.w_{n x}(x)\right|_{x=0}<0$, так как $w_{n}(x) \leqslant 0$ при $0<x<l$. Но это противоречит граничному условию $(21)$, левая часть которого строго отрицательна, в то время как правая часть $\left.h_{n}\right|_{x=0} K_{2} \tau n+\left.\left(1+K_{1} \tau\right)^{-n}\left(g_{n}+a_{n} \psi_{x}-h_{n} \psi\right)\right|_{x=0} \geqslant 0, n=\overline{1, N}$, в силу выбора постоянной $K_{3}$. Аналогичные рассуждения с использованием граничного условия $(22)$ позволяют установить, что $\left.w_{n}(x)\right|_{x=l} \leqslant 0$.

Наконец, из начального условия (23) и выбора постоянной $K_{3}$ в $(24)$ следует, что при $n=0 w_{0}(x) \leqslant 0$ при $0 \leqslant x \leqslant l$. Таким образом, всюду в области $\bar{Q}_{\tau}$ функция $w_{n}(x) \leqslant 0$, что вместе с $(14)$ и $(19)$ позволяет получить следующую оценку сверху для $u_{n}(x)$ :

$$
u_{n}(x) \leqslant\left(1+K_{1} \tau\right)^{n} K_{2} \tau n+\max _{0 \leqslant x \leqslant l}|\psi(x)| \leqslant K_{2} T \exp \left(K_{1} T\right)+K_{3} l\left(1+\frac{l}{4}\right) .
$$

Аналогичные рассуждения для вспомогательных функций $w_{n}(x)$ и $\psi(x)$ вида

$$
w_{n}(x)=v_{n}(x)+K_{2} \tau n, \quad \psi(x)=K_{3}\left(x-\frac{l}{2}\right)^{2}+K_{3} l
$$

приводят к оценке снизу для $u_{n}(x)$ :

$u_{n}(x)=\left(1+K_{1} \tau\right)^{n}\left(w_{n}(x)-K_{2} \tau n\right)-\psi(x) \geqslant-\left(1+K_{1} \tau\right)^{n} K_{2} \tau n-\max _{0 \leqslant x \leqslant l}|\psi(x)| \geqslant-K_{2} T \exp \left(K_{1} T\right)-K_{3} l\left(1+\frac{l}{4}\right)$,

что завершает доказательство леммы 1.

3.2.2. Оценка производной $u_{n x}$ в дифференциально-разностной краевой задаче (8)-(11). При получении априорной оценки для $u_{n x}$ в замкнутой области $\bar{Q}_{\tau}$ предположим сначала, что в исходной краевой задаче $(1)-(4) a=a(x, t), h(t, u)=0, e(t, u)=0$. Имеет место

Лемма 2. Пусть при $(x, t) \in \bar{Q}$ и любых $u,|u|<\infty$, коэффициенты и правая часть уравнения (1) являются ограниченными функциями в областях своего определения, причем коэфбициент $a(x, t)$ ограничен в $\bar{Q}$ вместе со своими производными по $x$ u $t$; кроме того, $0<a_{\min } \leqslant a \leqslant a_{\max }, 0<c_{\min } \leqslant c \leqslant c_{\max }$, $0<\rho_{\min } \leqslant \rho \leqslant \rho_{\max }, h=0, e=0$.

Предположим также, что функиии $\varphi(x), g(t)$ u $q(t)$ принадлежат, соответственно, пространствам $O^{1}[0, l]$ и $O^{1}[0, T]$ (т.е. ограничены вместе со своими производными) и удовлетворяют условиям согласования при $t=0:\left.a(x, 0) \varphi_{x}\right|_{x=0}=g(0),\left.a(x, 0) \varphi_{x}\right|_{x=l}=q(0)$.

Тогда в области $\bar{Q}_{\tau}$ при $\tau \leqslant \tau_{0}\left(\tau_{0}>0-\right.$ постоянная, определенная в лемме 1$)$ справедлива оценка производной решения дифференциально-разностной краевой задачи (8)-(11):

$$
\max _{\left(x, t_{n}\right) \in \bar{Q}_{\tau}}\left|u_{n x}(x)\right| \leqslant M_{1}
$$

где $M_{1}>0$ - постоянная, не зависящая от $x, \tau, n$.

Доказательство. Совершим замену

$$
\begin{gathered}
v_{n}(x)=u_{n}(x)-x^{2} \psi_{n}^{l}+(x-l)^{2} \psi_{n}^{0}, \quad\left(x, t_{n}\right) \in \bar{Q}_{\tau}, \\
\psi_{n}^{0}=g_{n}\left(\left.2 l a_{n}\right|_{x=0}\right)^{-1}, \quad \psi_{n}^{l}=q_{n}\left(\left.2 l a_{n}\right|_{x=l}\right)^{-1}, \quad n=\overline{1, N},
\end{gathered}
$$

сводящую граничные условия при $x=0$ и $x=l$ к однородным:

$$
v_{n x}(x)=u_{n x}(x)-2 x \psi_{n}^{l}+2(x-l) \psi_{n}^{0},\left.\quad v_{n x}(x)\right|_{x=0}=0,\left.\quad v_{n x}(x)\right|_{x=l}=0 .
$$

Продолжим четным образом функцию $v_{n}(x)$ в области $Q_{\tau}^{-}=\{-l<x<0\} \times \omega_{\tau}$ и $Q_{\tau}^{+}=\{l<x<2 l\} \times \omega_{\tau}$, полагая

$$
v_{n}(x)= \begin{cases}u_{n}(-x)-x^{2} \psi_{n}^{l}+(x+l)^{2} \psi_{n}^{0}, & \left(x, t_{n}\right) \in Q_{\tau}^{-}, \\ u_{n}(2 l-x)+(l-x)^{2} \psi_{n}^{0}-(2 l-x)^{2} \psi_{n}^{l}, & \left(x, t_{n}\right) \in Q_{\tau}^{+} .\end{cases}
$$

Построенная в области $Q_{\tau}^{0}=Q_{\tau}^{-} \cup \bar{Q}_{\tau} \cup Q_{\tau}^{+}$функция (за которой сохраним обозначение $v_{n}(x)$ ) непрерывна и обладает непрерывной производной $v_{n x}(x),\left.v_{n x}(x)\right|_{x=-l}=0,\left.v_{n x}(x)\right|_{x=2 l}=0, n=\overline{0, N}$. Имеет место соотношение

$$
c_{n} \rho_{n} v_{n \bar{t}}-\left(a_{n} v_{n x}\right)_{x}+b_{n} v_{n x}+d_{n} v_{n}=F_{n}^{0}(x), \quad\left(x, t_{n}\right) \in Q_{\tau}^{0}, \quad x \neq 0, \quad x \neq l
$$


в котором функции $a_{n}, b_{n}, c_{n}, d_{n}, \rho_{n}$ и $f_{n}$ продолжены четным образом в области $Q_{\tau}^{-}$и $Q_{\tau}^{+}$. Правая часть уравнения (27) имеет вид

$$
F_{n}^{0}(x)= \begin{cases}F_{n}(x), & \left(x, t_{n}\right) \in Q_{\tau}, \\ F_{n}^{-}(x), & \left(x, t_{n}\right) \in Q_{\tau}^{-}, \\ F_{n}^{+}(x), & \left(x, t_{n}\right) \in Q_{\tau}^{+},\end{cases}
$$

в котором приняты обозначения

$$
\begin{aligned}
F_{n}(x)= & f_{n}(x)+2 a_{n}\left(\psi_{n}^{l}-\psi_{n}^{0}\right)+2\left(a_{n x}-b_{n}\right)\left(x \psi_{n}^{l}-(x-l) \psi_{n}^{0}\right)- \\
& -c_{n} \rho_{n}\left(x^{2} \psi_{n \bar{t}}^{l}-(x-l)^{2} \psi_{n \bar{t}}^{0}\right)-d_{n}\left(x^{2} \psi_{n}^{l}-(x-l)^{2} \psi_{n}^{0}\right), \quad\left(x, t_{n}\right) \in Q_{\tau} \\
F_{n}^{-}(x)= & f_{n}(x)+2 a_{n}\left(\psi_{n}^{l}-\psi_{n}^{0}\right)+2\left(a_{n x}-b_{n}\right)\left(x \psi_{n}^{l}-(x+l) \psi_{n}^{0}\right)- \\
& -c_{n} \rho_{n}\left(x^{2} \psi_{n \bar{t}}^{l}-(x+l)^{2} \psi_{n \bar{t}}^{0}\right)-d_{n}\left(x^{2} \psi_{n}^{l}-(x+l)^{2} \psi_{n}^{0}\right), \quad\left(x, t_{n}\right) \in Q_{\tau}^{-} \\
F_{n}^{+}(x)= & f_{n}(x)+2 a_{n}\left(\psi_{n}^{l}-\psi_{n}^{0}\right)+2\left(a_{n x}-b_{n}\right)\left((x-2 l) \psi_{n}^{l}-(x-l) \psi_{n}^{0}\right)- \\
& -c_{n} \rho_{n}\left((2 l-x)^{2} \psi_{n \bar{t}}^{l}-(x-l)^{2} \psi_{n \bar{t}}^{0}\right)-d_{n}\left((2 l-x)^{2} \psi_{n}^{l}-(x-l)^{2} \psi_{n}^{0}\right), \quad\left(x, t_{n}\right) \in Q_{\tau}^{+} .
\end{aligned}
$$

Теперь воспользуемся подходом, разработанным в [4] для оценки производной решения первой краевой задачи для нелинейного параболического уравнения. Видоизменим этот подход применительно к дифференциально-разностной краевой задаче для $v_{n}(x)$ в области $Q_{\tau}^{0}$. Именно, введем дополнительную пространственную переменную $z$ и рассмотрим в области $\bar{\Pi}_{\tau}=\left\{\left(x, z, t_{n}\right):-l \leqslant z<x \leqslant 2 l, 0 \leqslant t_{n} \leqslant T\right\}$ функцию $W_{n}(x, z)=v_{n}(x)-v_{n}(z)$. Покажем, что при всех $\left(x, z, t_{n}\right) \in \bar{\Pi}_{\tau}$ имеет место оценка $\left|W_{n}(x, z)\right| \leqslant$ $\bar{M}_{1}|x-z|, \bar{M}_{1}=$ const $>0$, из которой следует оценка для $v_{n x}$, а из нее при условии существования производной $u_{n x}$ (см. (26)) следует и искомая оценка (25).

Прежде всего заметим, что $W_{n x}=v_{n x}(x), W_{n z}=-v_{n z}(z), W_{n x x}=v_{n x x}(x), W_{n z z}=-v_{n z z}(z)$, т.е. для $W_{n}(x, z)$ в силу $(27)$ справедливо следующее соотношение при $\left(x, z, t_{n}\right) \in \Pi_{\tau}, x, z \neq 0, x, z \neq l$ :

$$
\begin{aligned}
W_{n \bar{t}} & -\left(c_{n}(x) \rho_{n}(x)\right)^{-1}\left\{a_{n}(x) W_{n x x}-\left(b_{n}(x)-a_{n x}\right) W_{n x}\right\}- \\
& -\left(c_{n}(z) \rho_{n}(z)\right)^{-1}\left\{a_{n}(z) W_{n z z}-\left(b_{n}(z)-a_{n z}\right) W_{n z}\right\}= \\
& =\left(c_{n}(x) \rho_{n}(x)\right)^{-1}\left\{F^{0}(x)-d_{n}(x) v_{n}(x)\right\}-\left(c_{n}(z) \rho_{n}(z)\right)^{-1}\left\{F^{0}(z)-d_{n}(z) v_{n}(z)\right\} .
\end{aligned}
$$

Рассмотрим в $\bar{\Pi}_{\tau}$ вспомогательные функции

$$
w_{n}^{ \pm}(x, z)=\eta^{2}(x, z)\left\{\exp \left( \pm K_{4} W_{n}(x, z)\right)-1\right\}
$$

где $K_{4}>0$ - постоянная, выбираемая ниже, $\eta(x, z)=\zeta(x) \zeta(z), \zeta(x)-$ функция из $C^{2}[0, l]$, являющаяся "срезающей" для отрезка $[0, l]$ :

$$
\zeta(x)= \begin{cases}0, & -l \leqslant x \leqslant-l+\frac{\sigma}{2}, \\ 1, & -l+\sigma \leqslant x \leqslant 2 l-\sigma, \\ 0, & 2 l-\frac{\sigma}{2} \leqslant x \leqslant 2 l\end{cases}
$$

$\sigma>0$ - достаточно малая величина. Заметим, что в силу своего определения $\left.w_{n}^{ \pm}\right|_{x=z}=0,\left.w_{n}^{ \pm}\right|_{z=-l}=0$, $\left.w_{n}^{ \pm}\right|_{x=2 l}=0$ при $n=\overline{0, N}$. Образуем оператор $\mathcal{L} w_{n}^{ \pm}(x, z)$ вида

$$
\begin{aligned}
& \mathcal{L} w_{n}^{ \pm}(x, z) \equiv \mathcal{C} w_{n \bar{t}}^{ \pm}-\mathcal{A}_{n}(x) w_{n x x}^{ \pm}-\mathcal{A}_{n}(z) w_{n z z}^{ \pm}, \quad \text { где } \\
& \mathcal{A}_{n}(x)=\left(c_{n}(x) \rho_{n}(x)\right)^{-1} a_{n}(x) \exp \left(\mp K_{4} W_{n}\right), \quad \mathcal{C}_{n}=\left\{\int_{0}^{1} \exp \left( \pm K_{4}\left\{\theta W_{n}+(1-\theta) W_{n-1}\right\}\right) d \theta\right\}
\end{aligned}
$$


Из определения функции $w_{n}^{ \pm}$(см. (30)) следуют выражения для ее производных, в частности,

$$
\begin{aligned}
w_{n x}^{ \pm}= & \pm K_{4} W_{n x} \eta^{2}(x, z) \exp \left( \pm K_{4} W_{n}\right)+2 \eta \eta_{x}\left\{\exp \left( \pm K_{4} W_{n}\right)-1\right\}, \\
w_{n x x}^{ \pm}= & \left\{K_{4}^{2} W_{n x}^{2} \eta^{2}(x, z) \pm K_{4} W_{n x x} \eta^{2}(x, z) \pm 4 \eta \eta_{x}(x, z) K_{4} W_{n x}\right\} \exp \left( \pm K_{4} W_{n}\right)+ \\
& +\left\{2 \eta_{x}^{2}(x, z)+2 \eta \eta_{x x}(x, z)\right\}\left\{\exp \left( \pm K_{4} W_{n}\right)-1\right\}, \\
w_{n \bar{t}}^{ \pm}= & \pm K_{4} W_{n \bar{t}} \eta^{2}(x, z) \int_{0}^{1} \exp \left( \pm K_{4}\left\{\theta W_{n}+(1-\theta) W_{n-1}\right\}\right) d \theta .
\end{aligned}
$$

Учитывая эти соотношения и уравнение $(29)$ для $W_{n}(x, z)$, приходим к выводу, что

$$
\begin{gathered}
\mathcal{L} w_{n}^{ \pm}=\left(c_{n}(x) \rho_{n}(x)\right)^{-1}\left\{-K_{4}^{2} \eta^{2}(x, z) a_{n}(x) W_{n x}^{2} \pm K_{4} \eta^{2}(x, z)\left(a_{n x}(x)-b_{n}(x)\right) W_{n x} \mp\right. \\
\left.\mp 4 K_{4} \eta(x, z) \eta_{x}(x, z) a_{n}(x) W_{n x}\right\}+\left(c_{n}(z) \rho_{n}(z)\right)^{-1}\left\{-K_{4}^{2} \eta^{2}(x, z) a_{n}(z) W_{n z}^{2} \pm\right. \\
\left. \pm K_{4} \eta^{2}(x, z)\left(a_{n z}(z)-b_{n}(z)\right) W_{n z} \mp 4 K_{4} \eta(x, z) \eta_{z}(x, z) a_{n}(z) W_{n z}\right\}+\mathcal{F}_{n}(x)+\mathcal{F}_{n}(z), \\
\left(x, z, t_{n}\right) \in \Pi_{\tau}, \quad x, z \neq 0, \quad x, z \neq l .
\end{gathered}
$$

Функции $\mathcal{F}_{n}(x)$ и $\mathcal{F}_{n}(z)$ в правой части уравнения $(32)$ имеют вид

$$
\begin{aligned}
& \mathcal{F}_{n}(x)=\left(c_{n}(x) \rho_{n}(x)\right)^{-1}\left\{ \pm K_{4} \eta^{2}(x, z)\left(F_{n}^{0}(x)-d_{n}(x) v_{n}(x)\right)-2\left(\eta_{x}^{2}+\eta(x, z) \eta_{x x}\right) a_{n}(x)\left\{1-\exp \left(\mp K_{4} W_{n}\right)\right\}\right\}, \\
& \mathcal{F}_{n}(z)=\left(c_{n}(z) \rho_{n}(z)\right)^{-1}\left\{\mp K_{4} \eta^{2}(x, z)\left(F_{n}^{0}(z)-d_{n}(z) v_{n}(z)\right)-2\left(\eta_{z}^{2}+\eta(x, z) \eta_{z z}\right) a_{n}(z)\left\{1-\exp \left(\mp K_{4} W_{n}\right)\right\}\right\} .
\end{aligned}
$$

Применяя известное неравенство Коши с $\varepsilon, a b \leqslant \frac{\varepsilon}{2} a^{2}+\frac{1}{2 \varepsilon} b^{2}, \varepsilon>0-$ любое, заметим, что

$$
\begin{aligned}
& \pm K_{4} \eta^{2}(x, z)\left(a_{n x}(x)-b_{n}(x)\right) W_{n x} \leqslant K_{4} \eta^{2}(x, z) W_{n x}^{2}+\frac{1}{4} K_{4} \eta^{2}(x, z)\left(a_{n x}(x)-b_{n}(x)\right)^{2} \\
& \mp 4 K_{4} \eta(x, z) \eta_{x}(x, z) a_{n}(x) W_{n x} \leqslant K_{4} \eta^{2}(x, z) W_{n x}^{2}+4 K_{4} \eta_{x}^{2}(x, z) a_{n}^{2}(x) .
\end{aligned}
$$

Преобразуем таким же образом соответствующие члены с $W_{n z}$ в правой части (32) и затем выберем постоянную $K_{4}$ из условия $K_{4} \geqslant 2 a_{\mathrm{min}}^{-1}$. Это условие обеспечивает неотрицательность коэффициентов при $W_{n x}^{2}$ и $W_{n z}^{2}$, что позволяет отбросить эти слагаемые и установить неравенство

$$
\mathcal{L} w_{n}^{ \pm}(x, z) \leqslant 2 \max _{\left(x, z, t_{n}\right) \in \bar{\Pi}_{\tau}}\left|\mathcal{F}_{n}\right|, \quad\left(x, z, t_{n}\right) \in \Pi_{\tau}, \quad x, z \neq 0, \quad x, z \neq l .
$$

Заметим сразу, что функции $\mathcal{F}_{n}(x)$ и $\mathcal{F}_{n}(z)$ равномерно ограничены в области $\bar{\Pi}_{\tau}: \max _{\left(x, z, t_{n}\right) \in \bar{\Pi}_{\tau}}\left|\mathcal{F}_{n}\right| \leqslant K_{5}$, $K_{5}=$ const $>0$. Это следует из их вида и, в частности, из определения (28) функций $F_{n}^{0}$, причем постоянная $K_{5}$ очевидным образом зависит от величин, ограничивающих входные данные краевой задачи (1)-(4). Кроме того, $K_{5}$ зависит от величины $\bar{M}_{0}=\max _{\left(x, t_{n}\right) \in \bar{Q}_{\tau}}\left|v_{n}(x)\right|$ и, следовательно, в силу (26) от оценки (13). Особо отметим зависимость $K_{5}$ от $\max _{t_{n} \in \omega_{\tau}}\left|\psi_{n \bar{t}}^{0}\right|$ и $\max _{t_{n} \in \omega_{\tau}}\left|\psi_{n \bar{t}}^{l}\right|$, т.е. от величин $a_{t} \max , g_{t} \max , q_{t} \max ($ см. (26), $(28)$ и вид функций $\left.F_{n}, F_{n}^{-}, F_{n}^{+}\right)$.

Введем теперь в $\bar{\Pi}_{\tau}$ дополнительно вспомогательные функции

$$
v_{n}^{ \pm}(x, z)=w_{n}^{ \pm}(x, z)+K_{6}\{\exp (z-x)-1\}, \quad K_{6}=\text { const }>0,
$$

и покажем, что при соответствующем выборе $K_{6}$ функции $v_{n}^{ \pm}(x, z)<0$ всюду в $\bar{\Pi}_{\tau}$ кроме $x=z$, где $\left.v_{n}^{ \pm}\right|_{x=z}=0$. Заметим прежде всего, что в силу (30) и (33)

$$
\mathcal{L} v_{n}^{ \pm}(x, z)=\mathcal{L} w_{n}^{ \pm}(x, z)-K_{6}\left(\mathcal{A}_{n}(x)+\mathcal{A}_{n}(z)\right) \exp (z-x), \quad\left(x, z, t_{n}\right) \in \Pi_{\tau}, \quad x, z \neq 0, \quad x, z \neq l,
$$

т.е. при $K_{6}>K_{7}=K_{5} a_{\min }^{-1} c_{\max } \rho_{\max } \exp \left(2 K_{4} \bar{M}_{0}+3 l\right)$ имеет место неравенство

$$
\mathcal{L} v_{n}^{ \pm}(x, z)<0, \quad-l<z<x<2 l, 0<t_{n} \leqslant T .
$$


Кроме того, функции $v_{n}^{ \pm}(x, z)$ удовлетворяют условиям

$$
\left.v_{n}^{ \pm}\right|_{x=z}=0,\left.\quad v_{n}^{ \pm}\right|_{z=-l, z \neq x}<0,\left.\quad v_{n}^{ \pm}\right|_{x=2 l, x \neq z}<0, \quad n=\overline{0, N}
$$

которые вытекают из вида функций $w_{n}^{ \pm}(x, z)$ и $v_{n}^{ \pm}(x, z)($ см. $(30),(31)$ и $(34))$.

Исследуем теперь функцию $v_{n}^{ \pm}(x, z)$ при $n=0$ и выберем постоянную $K_{6}$ таким образом, чтобы

$$
v_{0 x}^{ \pm}(x, z)<0 \quad \text { при } \quad-l<z<x, \quad v_{0 z}^{ \pm}(x, z)>0 \quad \text { при } \quad z<x<2 l .
$$

Для этого рассмотрим выражения для $v_{0 x}^{ \pm}(x, z)$ и $v_{0 z}^{ \pm}(x, z)$ :

$$
\begin{aligned}
& v_{0 x}^{ \pm}(x, z)= \pm W_{0 x} \eta^{2}(x, z) \exp \left( \pm K_{4} W_{0}\right)+2 \eta(x, z) \eta_{x}(x, z)\left\{\exp \left( \pm K_{4} W_{0}\right)-1\right\}-K_{6} \exp (z-x), \\
& v_{0 z}^{ \pm}(x, z)= \pm W_{0 z} \eta^{2}(x, z) \exp \left( \pm K_{4} W_{0}\right)+2 \eta(x, z) \eta_{z}(x, z)\left\{\exp \left( \pm K_{4} W_{0}\right)-1\right\}+K_{6} \exp (z-x),
\end{aligned}
$$

и определим $K_{6}$ из условия $K_{6}>K_{8}=\left(\max _{0 \leqslant x \leqslant l}\left|W_{0 x}\right|+4 \max _{0 \leqslant x \leqslant l}\left|\eta_{x}\right| \bar{M}_{0}\right) \exp \left(2 K_{4} \bar{M}_{0}+3 l\right)$, в котором $\max _{0 \leqslant x \leqslant l}\left|W_{0 x}\right|>0$ - постоянная, зависящая в силу (26) от $\varphi_{x \max }, g_{\max }, q_{\max }, a_{\min }$. Такой выбор $K_{6}$ обеспечивает выполнение неравенства (37), а это вместе с условиями (36) при $n=0$ позволяет заключить, что $v_{0}^{ \pm}(x, z)<0$ при $-l \leqslant z<x \leqslant 2 l, t_{0}=0$.

Предположим, что на каждом временно́м слое $t=t_{j}$ при $j=\overline{1, n-1}$ неравенство $v_{j}^{ \pm}(x, z)<0$ выполняется при $-l \leqslant z<x \leqslant 2 l$. Доказательство того, что оно имеет место при $-l \leqslant z<x \leqslant 2 l$ и на слое $t=t_{n}$, основано на применении принципа максимума с учетом условий (35) и (36). Действительно, используя как и в [4] свойства равномерно эллиптических уравнений в граничных точках экстремума, заключаем, что $v_{n}^{ \pm}(x, z)<0$ при всех $(x, z):-l \leqslant z<x \leqslant 2 l$, в том числе и при $x=0, l, z=0, l$.

Из доказанного неравенства и из определений (30) и (34) функций $v_{n}^{ \pm}(x, z)$ и $w_{n}^{ \pm}(x, z)$ следует оценка для $W_{n}(x, z)=v_{n}(x)-v_{n}(z):\left|W_{n}(x, z)\right| \leqslant \bar{M}_{1}|x-z|, \bar{M}_{1}=K_{4}^{-1} K_{6} \exp \left(2 K_{4} \bar{M}_{0}\right), K_{6} \geqslant \max \left(K_{7}, K_{8}\right)$. Отсюда при условии существования производной $v_{n x}$ вытекает, что $\max _{\left(x, t_{n}\right) \in \bar{Q}_{\tau}}\left|v_{n x}(x)\right| \leqslant \bar{M}_{1}$. Это завершает доказательство леммы 2, так как из определения (26) сразу же следует и искомая оценка (25) для производной $u_{n x}$ с постоянной $M_{1}$, зависящей от $\bar{M}_{1}$ и от $g_{\max }, q_{\max }, a_{\min }$.

Замечание 1. Предложенный способ оценки производной $u_{n x}$ в случае граничных условий в исходной задаче вида

$$
\left.a(x, t) u_{x}\right|_{x=0}=g(t),\left.\quad a(x, t) u_{x}\right|_{x=l}=q(t), \quad 0<t \leqslant T,
$$

позволяет избежать дифференцирования уравнения (8) по переменной $x$ и, следовательно, не требует дополнительной гладкости входных данных. Однако этот способ нельзя применить для общей постановки задачи (1)-(4), т.е. при $a=a(x, t, u), h(t, u)>0, e(t, u)>0$. Это связано с тем, что функции $\psi_{n}^{0}$ и $\psi_{n}^{l}$, возникающие при сведении граничных условий (9) и (10) к однородным (см. (26)), принимают вид

$$
\psi_{n}^{0}=\left(g_{n}+\left.h_{n} u_{n}\right|_{x=0}\right)\left(\left.2 l a_{n}\right|_{x=0}\right)^{-1}, \quad \psi_{n}^{l}=\left(q_{n}-\left.e_{n} u_{n}\right|_{x=l}\right)\left(\left.2 l a_{n}\right|_{x=l}\right)^{-1} .
$$

Следовательно, в правую часть $F_{n}^{0}(x)$ уравнения $(27)$ войдут члены $\psi_{n \bar{t}}^{0}$ и $\psi_{n \bar{t}}^{l}$, содержащие $\left.u_{n \bar{t}}\right|_{x=0}$ и $\left.u_{n \bar{t}}\right|_{x=l}$, оценки которых неизвестны.

В общем случае устанавливает оценку производной $u_{n x}$ решения дифференциально-разностной краевой задачи (8)-(11)

Лемма 3. Пусть входные данные удовлетворяют условиям леммы (1) и, кроме того, при значениях $(x, t, u) \in \bar{D}=Q \times\left[-M_{0}, M_{0}\right]\left(M_{0}>0\right.$ - постоянная из оценки (13)) коэфбициент $a(x, t, u)$ принадлежит $O^{2,0,2}(\bar{D})$, функиии $b(x, t, u), c(x, t, u) u d(x, t, u)$ принадлежат $O^{1,0,1}(\bar{D})$, функции $\rho(x, t) u f(x, t)$ ограничены в $\bar{Q}$ вместе со свочми производными по х. Предположим также, что начальная функция $\varphi(x)$ принадлежит $O^{1}[0, l]$ и удовлетворяет условиям согласования при $t=0$ :

$$
\left.a(x, 0, \varphi) \varphi_{x}\right|_{x=0}-\left.h(0, \varphi) \varphi\right|_{x=0}=g(0),\left.\quad a(x, 0, \varphi) \varphi_{x}\right|_{x=l}+\left.e(0, \varphi) \varphi_{x}\right|_{x=l}=q(0) .
$$

Тогда в области $\bar{Q}_{\tau}$ при любом $\tau \leqslant \tau_{0}\left(\tau_{0}>0-\right.$ постоянная, определенная в лемме 1$)$ справедлива оценка производной решения дифференциально-разностной краевой задачи (8)-(11)

$$
\max _{\left(x, t_{n}\right) \in \bar{Q}_{\tau}}\left|u_{n x}(x)\right| \leqslant M_{1}
$$


в которой $M_{1}>0$ - постоянная, не зависящая от $x, \tau, n$.

Доказательство. Для получения оценки (38) используется известный способ, ограничимся его кратким изложением. Следуя, например, [1], вводится новая функция $v_{n}(x)$ с помощью нелинейной замены $u_{n}(x)=\Phi\left(v_{n}(x)\right)$, где $\Phi(v)$ - некоторая функция, ограниченная вместе со своими производными $\Phi_{v}(v)$, $\Phi_{v v}(v), \Phi_{v v v}(v)$ и удовлетворяющая условиям $\Phi_{v}(v)>0, \Phi_{v v}(v)<0, \Phi_{v v v}(v)<0$. При этом замена $\Phi(v)$ ставит в однозначное соответствие каждой функции $u_{n} \in\left[-M_{0}, M_{0}\right]$ функцию $v_{n}$ из интервала $(-1,1)$.

Затем уравнение (8) дифференцируется по $x$ с последующей подстановкой выражений $u_{n x}(x), u_{n x x}(x)$, $u_{n x x x}(x), u_{n x \bar{t}}(x)$ через $v_{n x}(x), v_{n x x}(x), v_{n x x x}(x), v_{n x \bar{t}}(x)$ с использованием указанной замены, в частности

$$
\begin{gathered}
u_{n x}(x)=\Phi_{v} v_{n x}(x), \quad u_{n x x}(x)=\Phi_{v} v_{n x x}(x)+\Phi_{v v} v_{n x}^{2}(x), \\
u_{n x x x}(x)=\Phi_{v} v_{n x x x}(x)+3 \Phi_{v v} v_{n x}(x) v_{n x x}(x)+\Phi_{v v v} v_{n x}^{3}(x) .
\end{gathered}
$$

Если ввести обозначение $w_{n}(x)=v_{n x}(x)$, то для этой функции после приведения подобных членов имеет место соотношение

$$
\Phi_{v} w_{n \bar{t}}-\left(c_{n} \rho_{n}\right)^{-1} a_{n} \Phi_{v} w_{n x x}+C_{n}^{0} w_{n x} w_{n}+C_{n}^{1} w_{n x}+C_{n}^{2} w_{n}^{3}+C_{n}^{3} w_{n}^{2}+C_{n}^{4} w_{n}+C_{n}^{5}=0, \quad\left(x, t_{n}\right) \in Q_{\tau},
$$

в котором все коэффициенты, в том числе $C_{n}^{i}, i=\overline{0,5}$, равномерно ограничены в области своего определения в силу условий леммы 3 и свойств $\Phi(v)$.

Завершающий этап доказательства состоит в построении такой функции $\Phi(v)$, которая кроме обладания указанными свойствами обеспечивает возможность применения принципа максимума к уравнению (39) с граничными условиями первого рода для $w_{n}(x)$. Получение оценки $\max _{\left(x, t_{n}\right) \in \bar{Q}_{\tau}}\left|w_{n}(x)\right|$ позволяет установить и искомую оценку для производной $u_{n x}(x)$.

3.2.3. Оценки норм в сеточно-непрерывных классах Гельдера для $u_{n}(x)$ и $u_{n x}(x)$. Получение этих оценок является следующим шагом в доказательстве разрешимости дифференциально-разностной краевой задачи (8)-(11). Их вывод основан на принадлежности $u_{n}(x)$ и $u_{n x}(x)$ (для которых установлены оценки $\left.\max _{\left(x, t_{n}\right) \in \bar{Q}_{\tau}}\left|u_{n}(x)\right| \leqslant M_{0}, \max _{\left(x, t_{n}\right) \in \bar{Q}_{\tau}}\left|u_{n x}(x)\right| \leqslant M_{1}\right)$ некоторым специальным классам $\mathcal{B}_{2 \tau}$ сеточнонепрерывных функций, вложимых в пространства Гельдера $H_{\tau}^{\lambda, \lambda / 2}$. Эти классы, введенные в [2, 3] как дискретные аналоги известных классов $\mathcal{B}_{2}$ из [1], определяются следующим образом:

$\mathcal{B}_{2 \tau}\left(\bar{Q}_{\tau}, \mathcal{M}, \nu, \varpi, \delta\right)-$ множество функций $w_{n}(x)$, принадлежащих $W_{2}^{1}[0, l]$ в любом фиксированном узле $t_{n}$ сетки $\omega_{\tau} \in[0, T]$, удовлетворяющих условию $\operatorname{vraimax}\left|w_{n}(x)\right| \leqslant \mathcal{M}$ и неравенствам $\left(x, t_{n}\right) \in \bar{Q}_{\tau}$

$$
\left(\int_{A_{k, r}\left(t_{n}\right)}\left(w_{n}-k\right)^{2} \zeta^{2}(x) d x\right)_{\bar{t}}+\nu \int_{A_{k, r}\left(t_{n}\right)} w_{n x}^{2} \zeta^{2}(x) d x \leqslant \varpi\left\{\int_{A_{k, r}\left(t_{n}\right)}\left(w_{n}-k\right)^{2} \zeta_{x}^{2} d x+\operatorname{meas} A_{k, r}\left(t_{n}\right)\right\}
$$

при всех $k$, для которых $k \geqslant \max \left\{\max _{x \in K_{r} \cap[0, l]} w_{n}(x)-\delta, w_{n}(0), w_{n}(l)\right\}$, и неравенствам

$$
\left(\int_{B_{k, r}\left(t_{n}\right)}\left(w_{n}-k\right)^{2} \zeta^{2}(x) d x\right)_{\bar{t}}+\nu \int_{B_{k, r}\left(t_{n}\right)} w_{n x}^{2} \zeta^{2}(x) d x \leqslant \varpi\left\{\int_{B_{k, r}\left(t_{n}\right)}\left(w_{n}-k\right)^{2} \zeta_{x}^{2} d x+\operatorname{meas} B_{k, r}\left(t_{n}\right)\right\}
$$

при всех $k$, для которых $k \leqslant \min \left\{\min _{x \in K_{r} \cap[0, l]} w_{n}(x)+\delta, w_{n}(0), w_{n}(l)\right\}$. Здесь $\zeta(x)$ - “срезающая" функция для интервала $K_{r}=\left\{x:\left|x-x_{0}\right| \leqslant r\right\}, 0<r<l, x_{0}$ - произвольная точка из отрезка $[0, l]$,

$$
A_{k, r}\left(t_{n}\right)=\left\{x: x \in K_{r} \cap[0, l], w_{n}(x)>k\right\}, \quad B_{k, r}\left(t_{n}\right)=\left\{x: x \in K_{r} \cap[0, l], w_{n}(x)<k\right\},
$$

параметры $\nu, \varpi, \delta$ - положительные константы, не зависящие от $\tau$ и $n$.

Вложимость $\mathcal{B}_{2 \tau}\left(\bar{Q}_{\tau}, \mathcal{M}, \nu, \varpi, \delta\right)$ в сеточно-непрерывный класс Гельдера $H_{\tau}^{\lambda, \lambda / 2}$ означает, что для любой функции $w_{n}(x)$ из этого множества в произвольном прямоугольнике

$$
Q_{\tau}^{\sigma_{1}, \sigma_{2}, \sigma_{3}}=\left\{\sigma_{1} \leqslant x \leqslant l-\sigma_{2}, \sigma_{3} \leqslant t_{n} \leqslant T\right\}, \quad \sigma_{i}>0, \quad i=1,2,3,
$$


имеет место оценка $\left\langle w_{n}(x)\right\rangle_{Q_{\tau}^{\sigma}, \sigma_{2}, \sigma_{3}}^{\lambda, \lambda / 2} \leqslant K$, в которой постоянные $K>0$ и $\lambda>0$ зависят только от параметров, определяющих класс $\mathcal{B}_{2 \tau}$. При $\sigma_{i}=0$ эта оценка зависит еще от констант Гельдера функции $w_{n}(x)$ при $x=0, x=l$ и при $n=0$. Доказательство этих утверждений аналогично доказательству соответствующих утверждений в [1] относительно классов $\mathcal{B}_{2}$.

Как установлено в $[2,3]$, решения $u_{n}(x)$ дифференциально-разностного уравнения вида

$$
u_{n \bar{t}}-\left(A_{n}\left(x, u_{n}\right) u_{n x}\right)_{x}+B_{n}\left(x, u_{n}, u_{n x}\right)=0, \quad\left(x, t_{n}\right) \in Q_{\tau},
$$

для которых $\max _{\left(x, t_{n}\right) \in \bar{Q}_{\tau}}\left|u_{n}(x)\right| \leqslant \mathcal{M}$, принадлежат классу $\mathcal{B}_{2 \tau}\left(\bar{Q}_{\tau}, \mathcal{M}, \nu, \varpi, \delta\right)$ с параметрами $\nu, \varpi, \delta$, определяемыми лишь значениями $\nu_{1}$ и $\mu_{1}$, где $\nu_{1}>0, \mu_{1}>0-$ постоянные, такие, что $\nu_{1} \leqslant A_{n}(x, u) \leqslant \mu_{1}$, $\left|B_{n}(x, u, p)\right| \leqslant \mu_{1}\left(1+|p|^{2}\right)$ при $\left(x, t_{n}\right) \in \bar{Q}_{\tau}$ и любых $u, p$.

Если для решений этого уравнения имеют место оценки $\max _{\left(x, t_{n}\right) \in \bar{Q}_{\tau}}\left|u_{n x}(x)\right| \leqslant \mathcal{M}_{1}, \mathcal{M}_{1}=$ const $>0$, то при выполнении условия

$$
\left|A_{n x}(x, u), A_{n u}(x, u), B_{n}(x, u, p)\right| \leqslant \mu_{2}, \quad \mu_{2}=\text { const }>0, \quad\left(x, t_{n}\right) \in \bar{Q}_{\tau}, \quad|u| \leqslant \mathcal{M}, \quad|p| \leqslant \mathcal{M}_{1},
$$

производные $u_{n x}(x)$ принадлежат классу $\mathcal{B}_{2 \tau}\left(\bar{Q}_{\tau}, \mathcal{M}_{1}, \nu, \varpi, \infty\right)$, в котором параметры $\nu$ и $\varpi$ определяются лишь величинами $\mathcal{M}, \mathcal{M}_{1}, \nu_{1}, \mu_{1}$ и $\mu_{2}$.

Возможность применить эти утверждения к рассматриваемой дифференциально-разностной краевой задаче (8)-(11) основана на том, что уравнение (8) принимает требуемый вид при

$A_{n}=a_{n}\left(c_{n} \rho_{n}\right)^{-1}, \quad B_{n}=\left(a_{n} c_{n}^{-1} \rho_{n}^{-1}\right)_{x} u_{n x}+\left(a_{n} c_{n}^{-1} \rho_{n}^{-1}\right)_{u} u_{n x}^{2}+\left(c_{n} \rho_{n}\right)^{-1}\left\{\left(b_{n}-a_{n x}\right) u_{n x}-a_{n u} u_{n x}^{2}+d_{n} u_{n}-f_{n}\right\}$.

Соответствующие оценки норм Гельдера в $H_{\tau}^{\lambda, \lambda / 2}$ для $u_{n}(x)$ и $u_{n x}(x)$ в случае входных данных $a=a(x, t)$, $h(t, u)=0, e(t, u)=0$ устанавливает

Лемма 4. Пусть выполнены условия леммы 2 и, кроме того, функиии $c(x, t, u)$ u $\rho(x, t)$ принадлежат, соответственно, $O^{1,0,1}(\bar{D})$ и $O^{1,0}(\bar{Q})$. Тогда для решения $u_{n}(x)$ задачи $(8)-(11)$ и его производной $u_{n x}(x)$ в произвольном прямоугольнике $Q_{\tau}^{\sigma_{1}, \sigma_{2}, \sigma_{3}}=\left\{\sigma_{1} \leqslant x \leqslant l-\sigma_{2}, \sigma_{3} \leqslant t_{n} \leqslant T\right\} \in \bar{Q}_{\tau}$ имеютместо оценки

$$
|\hat{u}(x)|_{Q_{\tau}^{\sigma_{1}, \sigma_{2}, \sigma_{3}}}^{\lambda, \lambda / 2} \leqslant M_{2}, \quad\left|\hat{u}_{x}(x)\right|_{Q_{\tau}^{\sigma_{1}, \sigma_{2}, \sigma_{3}}}^{\lambda, \lambda / 2} \leqslant M_{3},
$$

в которых постоянные $M_{2}>0, M_{3}>0$ и $\lambda>0$ определяются лишь параметрами соответствуюших классов $\mathcal{B}_{2 \tau}$ и значениями $\sigma_{i}$ при $\sigma_{i}>0(i=1,2,3)$.

Замечание 2. При $\sigma_{i}=0$ величины $M_{2}>0, M_{3}>0$ и $\lambda>0$ зависят еще от соответствующих начальных и граничных функций. Так, при $\sigma_{3}=0$ величины $M_{2}>0, M_{3}>0$ и $\lambda>0$ зависят еще от норм Гельдера $|\varphi(x)|_{[0, l]}^{\varepsilon}$ и $\left|\varphi_{x}(x)\right|_{[0, l]}^{\varepsilon}$. В случае $\sigma_{1}=0$ и $\sigma_{2}=0$ возникает, например, еще зависимость величины $M_{3}$ от оценки $\max _{0 \leqslant t_{n} \leqslant T}\left(\left|u_{n x \bar{t}}(0)\right|,\left|u_{n x \bar{t}}(l)\right|\right)$, т.е., в частности, от $g_{t} \max , q_{t} \max$. Это следует из вида граничных условий $\left.a(x, t) u_{x}(x)\right|_{x=0}=g(t),\left.a(x, t) u_{x}(x)\right|_{x=l}=q(t)$.

В общем случае при $a=a(x, t, u), h(t, u)>0, e(t, u)>0$ аналогичные оценки $(40)$ в $H_{\tau}^{\lambda, \lambda / 2}$ для $u_{n}(x)$ и $u_{n x}(x)$ справедливы при выполнении входными данными условий леммы 3. Соответствующие прямоугольники $Q_{\tau}^{\sigma_{1}, \sigma_{2}, \sigma_{3}}$ рассматриваются при $\sigma_{1}>0, \sigma_{2}>0$ и $\sigma_{3} \geqslant 0$, причем при $\sigma_{3}=0$ возникает зависимость величин $M_{2}, M_{3}$ и $\lambda$ от норм Гельдера $|\varphi(x)|_{[0, l]}^{1+\varepsilon}$.

3.2.4. Однозначная разрешимость дифференциально-разностной краевой задачи (8)-(11). Сформулируем теоремы существования и единственности решения этой задачи для входных данных $a=$ $a(x, t), h(t, u)=0, e(t, u)=0$, а также для общего случая $a=a(x, t, u), h(t, u)>0, e(t, u)>0$.

Теорема 1. Предположим, что:

1) при $(x, t) \in \bar{Q}$ и любых $u,|u|<\infty$, все входные данные задачи (1)-(4) являются ограниченными функииями в областях своего определения, причем коэффициент $а(x, t)$ ограничен в $\bar{Q}$ вместе со своими производными по $x$ и $t$; кроме того, $0<a_{\min } \leqslant a \leqslant a_{\max }, 0<c_{\min } \leqslant c \leqslant c_{\max }, 0<\rho_{\min } \leqslant \rho \leqslant \rho_{\max }, h=0$, $e=0$;

2) при $(x, t) \in \bar{Q}$ функиии $a_{x}(x, t), \rho(x, t)$ u $f(x, t)$ принадлежат $H^{\lambda, \lambda / 2}(\bar{Q})$, кроме того, $\rho(x, t)$ имеет ограниченную производную по $x$;

3) при $(x, t, u) \in \bar{D}=Q \times\left[-M_{0}, M_{0}\right]\left(M_{0}>0-\right.$ постоянная из оценки (13)) функции $b(x, t, u)$, $c(x, t, u)$ u d $(x, t, u)$ непрерывны в смысле Гельдера по $x$ и $t$ с показателями $\lambda, \lambda / 2$ и имеют ограниченные производные по и; кроме того, $c(x, t, u)$ имеет ограниченную производную по $x$; 
4) функиии $\varphi(x), g(t)$ и $q(t)$ принадлежат, соответственно, пространствам $H^{2+\lambda}[0, l] u O^{1}[0, T] u$ удовлетворяют условиям согласования при $t=0:\left.a(x, 0) \varphi_{x}\right|_{x=0}=g(0),\left.a(x, 0) \varphi_{x}\right|_{x=l}=q(0)$.

Тогда в области $\bar{Q}_{\tau}$ при любом $\tau \leqslant \tau_{0}\left(\tau_{0}>0-\right.$ постоянная, определенная в лемме 1$)$ существует единственное решение $u_{n}(x)$ дифференциально-разностной краевой задачи (8)-(11) и для него справедлива оценка

$$
|\hat{u}(x)|_{\bar{Q}_{\tau}}^{2+\lambda, 1+\lambda / 2} \leqslant M_{4}
$$

где $M_{4}>0$ - постоянная, не зависящая от $x, \tau, n$.

Теорема 2. Предположим, что:

1) при $(x, t) \in \bar{Q}$ и любых $u,|u|<\infty$, все входные данные задачи (1)-(4) являются ограниченными функциями в областях своего определения, причем коэфбициент $a(x, t, u)$ ограничен в $\bar{Q}$ вместе со свочми производными по $x$ u $u$; выполнены условия $0<a_{\min } \leqslant a \leqslant a_{\max }, 0<c_{\min } \leqslant c \leqslant c_{\max }$, $0<\rho_{\min } \leqslant \rho \leqslant \rho_{\max }, h(t, u) \geqslant 0, e(t, u) \geqslant 0$;

2) при $(x, t) \in \bar{Q}$ функции $\rho(x, t)$ и $f(x, t)$ имеют ограниченные производные по $x$ и непрерывны в смысле Гельдера по т с показателем $\lambda / 2$;

3) при $(x, t, u) \in \bar{D}=Q \times\left[-M_{0}, M_{0}\right]\left(M_{0}>0-\right.$ постоянная из оченки (13)) функции $a(x, t, u)$, $a_{x}(x, t, u), a_{u}(x, t, u), b(x, t, u), c(x, t, u) u d(x, t, u)$ принадлежат $H^{1, \lambda / 2,1}(\bar{D})$, функции $e(t, u) u h(t, u)$ имеют ограниченные производные по $t u u$;

4) функиии $\varphi(x), g(t)$ и $q(t)$ принадлежат, соответственно, пространствам $H^{2+\lambda}[0, l] u O^{1}[0, T]$, выполнены условия согласования при $t=0$ :

$$
\left.a(x, 0, \varphi) \varphi_{x}\right|_{x=0}-\left.h(0, \varphi) \varphi\right|_{x=0}=g(0),\left.\quad a(x, 0, \varphi) \varphi_{x}\right|_{x=l}+\left.e(0, \varphi) \varphi_{x}\right|_{x=l}=q(0) .
$$

Тогда в области $\bar{Q}_{\tau}$ при любом $\tau \leqslant \tau_{0}\left(\tau_{0}>0-\right.$ постоянная, определенная в лемме 1$)$ дифференциально-разностная краевая задача (8)-(11) имеет решение $u_{n}(x)$ в классе $H_{\tau}^{2+\lambda, 1+\lambda / 2}\left(Q_{\tau}\right)$, для которого справедлива оченка

$$
|\hat{u}(x)|_{Q_{\tau}}^{2+\lambda, 1+\lambda / 2} \leqslant M_{5}
$$

где $M_{5}>0$ - постоянная, не зависящая от $x, \tau, n$.

Это решение является единственным при выполнении условий

$$
h_{u \max } M_{0}+a_{u \max } M_{1} \leqslant h_{\min }, \quad e_{u \max } M_{0}+a_{u \max } M_{1} \leqslant e_{\min },
$$

где $M_{1}>0$ - постоянная из оценки (38).

Доказательство теорем 1, 2 основано на применении принципа Лерэ-Шаудера о существовании неподвижных точек вполне непрерывных преобразований (его формулировку см., например, в [1, 5]). Такой подход требует, кроме уже полученных априорных оценок в $H_{\tau}^{\lambda, \lambda / 2}$ для решения $u_{n}(x)$ и его производной $u_{n x}(x)$ в дифференциально-разностной краевой задаче (8)-(11), еще априорных оценок в $H_{\tau}^{2+\lambda, 1+\lambda / 2}$ решений краевой задачи для дифференциально-разностного аналога линейного параболического уравнения

$$
\begin{gathered}
\mathcal{L}_{n} u_{n} \equiv u_{n \bar{t}}-\mathcal{A}_{n}(x) u_{n x x}+\mathcal{B}_{n}(x) u_{n x}+\mathcal{C}_{n}(x) u_{n}=\mathcal{F}_{n}(x), \quad\left(x, t_{n}\right) \in Q_{\tau}, \\
\left.u_{n x}\right|_{x=0}=g_{n},\left.\quad u_{n x}\right|_{x=l}=q_{n}, \quad 0<t_{n} \leqslant T, \quad u_{0}(x)=\varphi(x), \quad 0 \leqslant x \leqslant l .
\end{gathered}
$$

Такие оценки, установленные в $[2,3]$ при условии принадлежности $\mathcal{A}_{n}(x), \mathcal{B}_{n}(x), \mathcal{C}_{n}(x)$ и $\mathcal{F}_{n}(x)$ классу Гельдера $H_{\tau}^{\lambda, \lambda / 2}$ и соответствующей принадлежности $\varphi(x), g_{n}$ и $q_{n}$ классам $H^{2+\lambda}[0, l]$ и $O_{\tau}^{1}[0, T]$, имеют вид

$$
|\hat{u}(x)|_{\bar{Q}_{\tau}}^{2+\lambda, 1+\lambda / 2} \leqslant K\left\{|\hat{\mathcal{F}}(x)|_{\bar{Q}_{\tau}}^{\lambda, \lambda / 2}+|\varphi(x)|_{[0, l]}^{2+\lambda}+|\hat{g}|_{\bar{\omega}_{\tau}}^{1}+|\hat{q}|_{\bar{\omega}_{\tau}}^{1}\right\}
$$

постоянная $K>0$ определяется величинами $\lambda(0<\lambda<1), \max \left(|\hat{\mathcal{A}}(x)|_{\bar{Q}_{\tau}}^{\lambda, \lambda / 2},|\hat{\mathcal{B}}(x)|_{\bar{Q}_{\tau}}^{\lambda, \lambda / 2},|\hat{\mathcal{C}}(x)|_{\bar{Q}_{\tau}}^{\lambda, \lambda / 2}\right)$ и не зависит от $x, \tau, n$.

Ограничимся кратким изложением схемы доказательства теоремы 2. Доказательство теоремы 1 проводится по аналогичной схеме с соответствующими упрощениями.

Доказательство теоремы 2. Для любой функции $\omega_{n}(x) \in H_{\tau}^{1+\lambda, \frac{1+\lambda}{2}}\left(\bar{Q}_{\tau}\right)$ из выпуклого ограниченного множества

$$
\begin{aligned}
\bar{\Omega}=\{ & \omega_{n}(x) \in H_{\tau}^{1+\lambda,(1+\lambda) / 2}\left(\bar{Q}_{\tau}\right), \max _{\left(x, t_{n}\right) \in \bar{Q}_{\tau}}|\omega| \leqslant \bar{M}_{0}+\varepsilon, \\
& \left.\max _{\left(x, t_{n}\right) \in \bar{Q}_{\tau}}\left|\omega_{x}\right| \leqslant \bar{M}_{1}+\varepsilon, \quad|\omega| \bar{Q}_{\tau}^{\lambda, \lambda / 2}+\left|\omega_{x}\right| \bar{Q}_{\tau}{ }^{\lambda, \lambda / 2} \leqslant \bar{M}_{2}+\bar{M}_{3}+\varepsilon\right\}
\end{aligned}
$$


и для любого числа $\theta, 0 \leqslant \theta \leqslant 1$, обозначим через $v_{n}=\Phi\left(\omega_{n}, \theta\right)$ решение линейной дифференциальноразностной краевой задачи

$$
\begin{gathered}
\mathcal{L}_{n}^{\theta} v_{n} \equiv c_{n}(x, \omega) \rho_{n}(x) v_{n \bar{t}}-\mathcal{A}_{n}(x, \omega) v_{n x x}+\theta \mathcal{F}_{n}(x, \omega)=0, \quad\left(x, t_{n}\right) \in Q_{\tau}, \\
\mathcal{A}_{n}(x, \omega) v_{n x}-\left.\left\{\theta h_{n}\left(\omega_{n}\right)+(1-\theta) a_{\min }\right\} v_{n}(x)\right|_{x=0}=\theta g_{n}+\left.(1-\theta) a_{\min }\left(\varphi_{x}(x)-\varphi(x)\right)\right|_{x=0}, \quad 0<t_{n} \leqslant T, \\
\mathcal{A}_{n}(x, \omega) v_{n x}+\left.\left\{\theta e_{n}\left(\omega_{n}\right)+(1-\theta) a_{\min }\right\} v_{n}(x)\right|_{x=l}=\theta q_{n}+\left.(1-\theta) a_{\min }\left(\varphi_{x}(x)+\varphi(x)\right)\right|_{x=l}, \quad 0<t_{n} \leqslant T, \\
v_{0}(x)=\varphi(x), \quad 0 \leqslant x \leqslant l, \\
\text { где }\left\{\begin{array}{l}
\mathcal{A}_{n}(x, \omega)=\theta a\left(x, t_{n}, \omega_{n}\right)+(1-\theta) a_{\min }, \\
\mathcal{F}_{n}(x, \omega)=\left\{b\left(x, t_{n}, \omega_{n}\right)-a_{x}\left(x, t_{n}, \omega_{n}\right)\right\} \omega_{n x}-a_{u}\left(x, t_{n}, \omega_{n}\right) \omega_{n x}^{2}+d\left(x, t_{n}, \omega_{n}\right) \omega_{n}-f\left(x, t_{n}\right), \\
c_{n}\left(x, \omega_{n}\right)=\theta c\left(x, t_{n}, \omega_{n}\right)+(1-\theta) c_{\min }, \quad \rho_{n}(x)=\rho\left(x, t_{n}\right), \\
h_{n}\left(\omega_{n}\right)=h\left(t_{n}, \omega_{n}\right), \quad e_{n}\left(\omega_{n}\right)=e\left(t_{n}, \omega_{n}\right), \quad g_{n}=g\left(t_{n}\right), \quad q_{n}=q\left(t_{n}\right) .
\end{array}\right.
\end{gathered}
$$

Возможность определения оператора $\Phi\left(\omega_{n}, \theta\right)$ следует из однозначной разрешимости задачи (45)-(48), которую можно рассматривать при каждом $n=\overline{1, N}$ как краевую задачу для линейного эллиптического уравнения с правой частью $c_{n}(x, \omega) \rho_{n}(x) \tau^{-1} v_{n-1}(x)-\theta \mathcal{F}_{n}(x, \omega)$ и коэффициентом $-c_{n}(x, \omega) \rho_{n}(x) \tau^{-1}$ при $v_{n}(x)$.

Неподвижные точки преобразования $\Phi\left(\omega_{n}, \theta\right)$, т.е. точки $u_{n}^{\theta}=\Phi\left(u_{n}^{\theta}, \theta\right)$, являются решениями квазилинейной дифференциально-разностной краевой задачи, для которой выполнены все условия применимости лемм 1, 3. Следовательно, справедливы оценки $\max _{\left(x, t_{n}\right) \in \bar{Q}_{\tau}}\left|u_{n}^{\theta}(x)\right| \leqslant \bar{M}_{0}, \max _{\left(x, t_{n}\right) \in \bar{Q}_{\tau}}\left|u_{n x}^{\theta}(x)\right| \leqslant \bar{M}_{1}$ и, соответственно, оценки (см. (40)) $\left|\hat{u}^{\theta}(x)\right|_{Q_{\tau}}^{\lambda, \lambda / 2} \leqslant \bar{M}_{2},\left|\hat{u}_{x}^{\theta}(x)\right|_{Q_{\tau}}^{\lambda, \lambda / 2} \leqslant \bar{M}_{3}$. Это означает, что все возможные неподвижные точки $u_{n}^{\theta}$ преобразования $\Phi\left(\omega_{n}, \theta\right)$ лежат строго внутри множества $\bar{\Omega}$. Заметим при этом, что при $\theta=1$ рассматриваемая задача (45)-(48) совпадает с задачей (8)-(11).

Определенный таким образом оператор $\Phi\left(\omega_{n}, \theta\right)$ является вполне непрерывным на $\bar{\Omega} \times[0,1]$, переводя множество $\bar{\Omega}$, ограниченное в $H_{\tau}^{1+\lambda,(1+\lambda) / 2}\left(Q_{\tau}\right)$, в компактное множество. Действительно, в силу оценок (44) решения $v_{n}(x)$ линейной дифференциально-разностной краевой задачи (45)-(48) равномерно ограничены в $H_{\tau}^{2+\lambda, 1+\lambda / 2}\left(Q_{\tau}\right)$ при любых $\omega_{n} \in \bar{\Omega}$ и $0 \leqslant \theta \leqslant 1$.

На основе оценок (44) нетрудно установить и равномерную непрерывность оператора $\Phi\left(\omega_{n}, \theta\right)$ на $\bar{\Omega} \times[0,1]$. Пусть $\omega_{n}^{(1)}(x)$ и $\omega_{n}^{(2)}(x)$ - два элемента множества $\bar{\Omega}$, близкие в норме $H_{\tau}^{1+\lambda,(1+\lambda) / 2}\left(Q_{\tau}\right)$. Тогда разность $\Delta v_{n}(x)=v_{n}^{(2)}(x)-v_{n}^{(1)}(x)$, где $v_{n}^{(2)}=\Phi\left(\omega_{n}^{(2)}, \theta\right), v_{n}^{(1)}=\Phi\left(\omega_{n}^{(1)}, \theta\right)$, мала в норме $H_{\tau}^{2+\lambda, 1+\lambda / 2}\left(Q_{\tau}\right)$. Это следует из оценок типа (44), примененных к $\Delta v_{n}(x)$ как к решению соответствующей линейной дифференциально-разностной краевой задачи.

Для возможности применения принципа Лерэ-Шаудера осталось показать, что при $\theta=0$ преобразование $\Phi\left(\omega_{n}, \theta\right)$ имеет внутри множества $\bar{\Omega}$ единственную неподвижную точку и оно обратимо в окрестности этой точки. Но это действительно имеет место, так как $\Phi\left(\omega_{n}, 0\right)$ переводит все множество $\bar{\Omega}$ в единственный элемент $v_{n}^{0}=\Phi\left(\omega_{n}, 0\right)$, являющийся решением линейной дифференциально-разностной краевой задачи

$$
\begin{gathered}
\mathcal{L}_{n}^{0} w_{n} \equiv c_{n}(x, \omega) \rho_{n}(x) v_{n \bar{t}}-a_{\min } v_{n x x}=0, \quad\left(x, t_{n}\right) \in Q_{\tau}, \\
a_{\min } v_{n x}(x)-\left.a_{\min } v_{n}(x)\right|_{x=0}=\left.a_{\min }\left(\varphi_{x}(x)-\varphi(x)\right)\right|_{x=0}, \quad 0<t_{n} \leqslant T, \\
a_{\min } v_{n x}(x)+\left.a_{\min } v_{n}(x)\right|_{x=l}=\left.a_{\min }\left(\varphi_{x}(x)+\varphi(x)\right)\right|_{x=l}, \quad 0<t_{n} \leqslant T, \\
w_{0}(x)=\varphi(x), \quad 0 \leqslant x \leqslant l .
\end{gathered}
$$

Эта задача, рассматриваемая при каждом $n=\overline{1, N}$ как краевая задача для линейного эллиптического уравнения, имеет и притом единственное (в силу коэффициента $-c_{n}(x, \omega) \rho_{n}(x) \tau^{-1}$ при $\left.v_{n}(x)\right)$ решение.

Таким образом, принцип Лерэ-Шаудера позволяет заключить, что при всех $\theta \in[0,1]$ (а следовательно, и при $\theta=1)$ существует по крайней мере одна неподвижная точка преобразования $\Phi\left(\omega_{n}, \theta\right)$, принадлежащая $H_{\tau}^{1+\lambda,(1+\lambda) / 2}\left(Q_{\tau}\right)$. Как уже было отмечено, такая точка при $\theta=1$ является решением краевой задачи (8)-(11).

Нетрудно показать, используя оценки (44), что это решение принадлежит классу $H_{\tau}^{2+\lambda, 1+\lambda / 2}\left(Q_{\tau}\right)$, так как коэффициенты уравнения (45) при подстановке в них $u_{n}^{\theta}(x)$ при $\theta=1$ вместо $\omega_{n}(x)$ являются как функции $(x, t)$ элементами соответствующих сеточно-непрерывных классов Гельдера. 
Так как по условиям теоремы 2 начальная функция $\varphi(x) \in H^{2+\lambda}[0, l]$, то решение $u_{n}(x)$ принадлежит указанному классу $H_{\tau}^{2+\lambda, 1+\lambda / 2}$ при всех $0<x<l, 0 \leqslant t_{n} \leqslant T$. При этом его производная $u_{n x}(x)$ непрерывна во всей замкнутой области $\bar{Q}_{\tau}$, т.е. $\lim _{x \rightarrow 0} u_{n x}(x)=u_{n x}(0), \lim _{x \rightarrow l} u_{n x}(x)=u_{n x}(l), n=\overline{1, N}$. Доказательство этого утверждения основано на классическом методе барьеров (см., например, [1]), примененном к уравнению $(39)$ для $w_{n}(x)$. Именно, рассматривается оператор

$$
\mathcal{L}_{n} w_{n} \equiv \Phi_{v} w_{n \bar{t}}-\left(c_{n} \rho_{n}\right)^{-1} a_{n} \Phi_{v} w_{n x x}+C_{n}^{0} w_{n x} w_{n}+C_{n}^{1} w_{n x}, \quad\left(x, t_{n}\right) \in Q_{\tau},
$$

для которого в силу равномерной ограниченности коэффициентов $C_{n}^{i}(i=\overline{0,5})$ в $(39)$ и полученных оценок $(38)$ следует неравенство $\mathcal{L}_{n} w_{n} \leqslant \mathcal{K}$ при $\left(x, t_{n}\right) \in Q_{\tau}$, где $\mathcal{K}>0$ - постоянная, зависящая, в частности, от $M_{1}$ (см. (38)) и не зависящая от $x, \tau, n$. Далее используется обычный прием построения барьерных функций для доказательства, того что $\lim _{x \rightarrow 0} w_{n}(x)=w_{n}(0), \lim _{x \rightarrow l} w_{n}(x)=w_{n}(l), n=\overline{1, N}$.

Для завершения доказательства теоремы 2 осталось установить единственность решения $u_{n}(x)$ краевой задачи (8)-(11) в классе функций $\sup _{\left(x, t_{n}\right) \in \bar{Q}}\left|u_{n}(x), u_{n x}(x), u_{n x x}(x), u_{n \bar{t}}(x)\right|<\infty$. Допустим, что $\bar{u}_{n}(x)$ тоже является решением. Тогда их разность $v_{n}(x)=u_{n}(x)-\bar{u}_{n}(x)$ удовлетворяет соотношениям

$$
\begin{gathered}
c_{n}\left(x, u_{n}\right) \rho_{n}(x) v_{n \bar{t}}-\left(a_{n}\left(x, u_{n}\right) v_{n x}\right)_{x}+\mathcal{A}_{n}^{0} v_{n x}+\mathcal{A}_{n}^{1} v_{n}=0, \quad\left(x, t_{n}\right) \in Q_{\tau}, \\
a_{n}\left(x, u_{n}\right) v_{n x}-\left.\mathcal{A}_{n}^{2} v_{n}\right|_{x=0}=0, \quad 0<t_{n} \leqslant T, \\
a_{n}\left(x, u_{n}\right) v_{n x}+\left.\mathcal{A}_{n}^{3} v_{n}\right|_{x=l}=0, \quad 0<t_{n} \leqslant T, \\
v_{0}(x)=0, \quad 0 \leqslant x \leqslant l,
\end{gathered}
$$

где коэффициенты $\mathcal{A}_{n}^{0}$ и $\mathcal{A}_{n}^{1}$ зависят от производных $a_{u}, a_{x u}, a_{u u}, b_{u}, c_{u}$ и $d_{u}$ в точке $\left(x, t_{n}, \sigma u_{n}+(1-\sigma) \bar{u}_{n}\right)$ $(0<\sigma<1)$, а также от решения $u_{n}(x)$ и его производных $u_{n x}(x), u_{n x x}(x)$ и $u_{n \bar{t}}(x)$. Коэффициенты $\mathcal{A}_{n}^{2}$ и $\mathcal{A}_{n}^{3}$ в граничных условиях зависят соответствующим образом от $a_{u}, h_{u}$ и $e_{u}$, причем $\mathcal{A}_{n}^{2} \geqslant 0, \mathcal{A}_{n}^{3} \geqslant 0$ при выполнении условия (43). Поскольку входные данные этой линейной дифференциально-разностной краевой задачи равномерно ограничены в $\bar{Q}_{\tau}$ как функции $\left(x, t_{n}\right)$, то применение принципа максимума позволяет заключить, что $\max _{\left(x, t_{n}\right) \in \bar{Q}_{\tau}}\left|v_{n}(x)\right|=0$. Теорема 2 доказана.

Замечание 3. Если входные данные дифференциально-разностной краевой задачи (8)-(11) удовлетворяют условиям теоремы 1 , то решение $u_{n}(x)$ принадлежит классу $H_{\tau}^{2+\lambda, 1+\lambda / 2}$ во всей замкнутой области $\bar{Q}_{\tau}$. Это связано с тем, что в данном случае, кроме априорной оценки $\max _{\left(x, t_{n}\right) \in \bar{Q}_{\tau}}\left|u_{n x}(x)\right| \leqslant M_{1}$, справедливы еще оценки приграничных производных $\max _{0 \leqslant t_{n} \leqslant T}\left|u_{n x \bar{t}}(x)\right|$ при $x=0$ и $x=l$. Это позволяет утверждать, что во всей замкнутой области имеет место оценка нормы Гельдера $|\hat{u}(x)|_{\bar{Q}_{\tau}}^{\lambda, \lambda / 2} \leqslant M_{3}$, а следовательно, и оценка $|\hat{u}(x)|_{\bar{Q}_{\tau}}^{2+\lambda, 1+\lambda / 2} \leqslant M_{4}$.

4. Существование и единственность решения $\{u(x, t), \rho(x, t)\}$ нелинейной параболической системы (1)-(5).

4.1. Доказательство разрешимости этой системы с помощью метода прямых Ротэ основано на исследовании нелинейной дифференциально-разностной системы (8)-(12) для определения $\left\{u_{n}(x), \rho_{n}(x)\right\}-$ приближенных значений функций $u(x, t)$ и $\rho(x, t)$ при $t=t_{n}$.

При решении системы $(8)-(12)$ сеточно-непрерывные функции $\rho_{n}(x)$ заранее не известны и ищутся совместно с $u_{n}(x)$, что требует дополнительных рассуждений при выводе априорных оценок и при доказательстве существования решения $\left\{u_{n}(x), \rho_{n}(x)\right\}$. Имеет место

Лемма 5. Пусть входные даннъе системы (1)-(5) удовлетворяют требованиям теоремы 2. Предположим также, что функция $\gamma(x, t, u)$ принадлежит пространству $H^{1, \lambda / 2,1}(\bar{D})$, начальная функция $\rho^{0}(x)$ в условии (5) принадлежит $C^{1}[0, l]$ и удовлетворяет неравенству $0<\rho_{\min }^{0} \leqslant \rho^{0}(x) \leqslant \rho_{\max }^{0}$, $\rho_{\min }^{0}, \rho_{\max }^{0}=$ const $>0$.

Тогда в области $\bar{Q}_{\tau}$ при любом $\tau \leqslant \tau_{0}\left(\tau_{0}>0-\right.$ постоянная, определенная в лемме 1$)$ дифференциально-разностная система (8)-(12) имеет единственное решение $\left\{u_{n}(x), \rho_{n}(x)\right\}$, такое, что

$$
u_{n}(x) \in H_{\tau}^{2+\lambda, 1+\lambda / 2}\left(Q_{\tau}\right), \quad|\hat{u}(x)|_{Q_{\tau}}^{2+\lambda, 1+\lambda / 2} \leqslant M_{5}, \quad M_{5}=\text { const }>0 \quad \text { из оценки }
$$


$\rho_{n}(x)$ - ограниченная функция, удовлетворяющая в зависимости от знака функции $\gamma(x, t, u)$ оценкам

$$
\begin{gathered}
0<\rho_{\min }^{0}<\rho_{n}(x) \leqslant \rho_{\max }, \quad \rho_{\max }=\rho_{\max }^{0}+T \max _{(x, t, u) \in \bar{D}} \gamma(x, t, u) \quad n p u \quad \gamma(x, t, u)>0, \quad(x, t, u) \in \bar{D} \\
0<\rho_{\min }^{0}-T \max _{(x, t, u) \in \bar{D}}|\gamma(x, t, u)| \leqslant \rho_{n}(x) \leqslant \rho_{\max }^{0} \quad n p u \quad \gamma(x, t, u) \leqslant 0, \quad(x, t, u) \in \bar{D}
\end{gathered}
$$

и обладающая ограниченными в $\bar{Q}_{\tau}$ производными $\rho_{n x}(x), \rho_{n \bar{t}}(x), \rho_{n x \bar{t}}(x)$ и величиной $\left\langle\hat{\rho}_{\bar{t}}(x)\right\rangle_{t, \bar{Q}_{\tau}}^{\lambda / 2}$ :

$$
\max _{\left(x, t_{n}\right) \in \bar{Q}_{\tau}}\left|\rho_{n x}(x)\right|+\max _{\left(x, t_{n}\right) \in \bar{Q}_{\tau}}\left|\rho_{n \bar{t}}(x)\right|+\max _{\left(x, t_{n}\right) \in \bar{Q}_{\tau}}\left|\rho_{n x \bar{t}}(x)\right|+\left\langle\hat{\rho}_{\bar{t}}(x)\right\rangle_{t, \bar{Q}_{\tau}}^{\lambda / 2} \leqslant \mathcal{M}
$$

$\mathcal{M}>0$ - постоянная, не зависящая от $x, \tau, n$ и определяемая, в частности, величинами $\max _{0 \leqslant x \leqslant l}\left|\rho_{x}^{0}(x)\right|$, $\max _{(x, t, u) \in \bar{D}}\left|\gamma_{x}(x, t, u)\right|, \max _{(x, t, u) \in \bar{D}}\left|\gamma_{u}(x, t, u)\right|$, а также величинами $M_{1}$ и $M_{2}$ из оченок (38), (40).

Доказательство. Исходя из начального момента времени $t_{0}=0$, предположим, что вплоть до момента $t=t_{n-1}$ решения $\left\{u_{j}(x), \rho_{j}(x)\right\}(j=\overline{1, n-1})$ уже найдены и соответствующие оценки для них уже установлены. Тогда при $t=t_{n}$ требования леммы 5 позволяют заключить из соотношения (12), что при $0 \leqslant x \leqslant l$

$$
\begin{gathered}
\left|\rho_{n \bar{t}}(x)\right| \leqslant \gamma_{\max }, \quad \gamma_{\max }=\max _{(x, t, u) \in \bar{D}}|\gamma(x, t, u)|, \quad\left\langle\hat{\rho}_{\bar{t}}(x)\right\rangle_{t, \bar{Q}_{\tau}}^{\lambda / 2} \leqslant\langle\gamma\rangle_{t, \bar{D}}^{\lambda / 2}+\max _{(x, t, u) \in \bar{D}}\left|\gamma_{u}(x, t, u)\right| M_{2}, \\
\left|\rho_{n x \bar{t}}(x)\right| \leqslant \max _{(x, t, u) \in \bar{D}}\left|\gamma_{x}(x, t, u)\right|+\max _{(x, t, u) \in \bar{D}}\left|\gamma_{u}(x, t, u)\right| M_{1} .
\end{gathered}
$$

Действительно, это очевидным образом следует из (12), так как

$$
\begin{gathered}
\left\langle\hat{\rho}_{\bar{t}}(x)\right\rangle_{t, \bar{Q}_{\tau}}^{\lambda / 2} \leqslant\left\langle\hat{\gamma}\left(x, t_{n-1}, u_{n-1}\right)\right\rangle_{t, \bar{D}_{\tau}}^{\lambda / 2}+\left|\gamma_{u}\left(x, t_{n-1}, u_{n-1}\right)\right| M_{2}, \\
\rho_{n x \bar{t}}(x)=\gamma_{x}\left(x, t_{n-1}, u_{n-1}\right)+\gamma_{u}\left(x, t_{n-1}, u_{n-1}\right) u_{n-1, x}(x) .
\end{gathered}
$$

Кроме того, из (12) следует, что

$$
\rho_{n}(x)=\rho_{n-1}(x)+\tau \gamma_{n-1}\left(x, u_{n-1}\right)=\rho^{0}(x)+\sum_{j=0}^{n-1} \tau \gamma_{j}\left(x, u_{j}\right),
$$

т.е. в зависимости от знака функции $\gamma(x, t, u)$ справедливы неравенства

$$
\begin{array}{llll}
0<\rho_{\min }^{0}<\rho_{n}(x) \leqslant \rho_{\max }^{0}+t_{n-1} \gamma_{\max } \quad \text { при } \quad \gamma(x, t, u)>0, & (x, t, u) \in \bar{D}, \\
0<\rho_{\min }^{0}-t_{n-1} \gamma_{\max } \leqslant \rho_{n}(x) \leqslant \rho_{\max }^{0} \quad \text { при } \quad \gamma(x, t, u) \leqslant 0, \quad(x, t, u) \in \bar{D} .
\end{array}
$$

Отсюда очевидным образом устанавливаются искомые оценки (49) леммы 5 для $\rho_{n}(x)$.

Заметим далее, что в силу (51) имеет место представление

$$
\rho_{n x}(x)=\rho_{x}^{0}(x)+\sum_{j=0}^{n-1} \tau\left\{\gamma_{x}\left(x, t_{j}, u_{j}\right)+\gamma_{u}\left(x, t_{j}, u_{j}\right) u_{j x}(x)\right\},
$$

из которого следует оценка

$$
\left|\rho_{n x}(x)\right| \leqslant \max _{0 \leqslant x \leqslant l}\left|\rho_{x}^{0}(x)\right|+t_{n-1}\left\{\max _{(x, t, u) \in \bar{D}}\left|\gamma_{x}(x, t, u)\right|+\max _{(x, t, u) \in \bar{D}}\left|\gamma_{u}(x, t, u)\right| M_{1}\right\} .
$$

Таким образом, при $t=t_{n}$ получены оценки для $\left|\rho_{n x}(x)\right|,\left|\rho_{n \bar{t}}(x)\right|,\left|\rho_{n x \bar{t}}(x)\right|$ и $\left\langle\hat{\rho}_{\bar{t}}(x)\right\rangle_{t, \bar{Q}_{\tau}}^{\lambda / 2}$. Это вместе с предположением, что соответствующие оценки в предыдущие моменты времени уже установлены, позволяет доказать оценку (50).

Следовательно, сеточно-непрерывная функция $\rho_{n}(x)$, найденная из условия $(12)$ по известным $\rho_{n-1}(x)$ и $u_{n-1}(x)$, удовлетворяет требованиям теоремы 2 относительно коэффициента $\rho_{n}(x)$ в уравнении $(8)$. В 
силу этой теоремы дифференциально-разностная краевая задача (8)-(11) имеет единственное решение $u_{n}(x)$ в классе $H_{\tau}^{2+\lambda, 1+\lambda / 2}\left(Q_{\tau}\right)$ и для него справедлива оценка (42). Лемма 5 доказана.

Замечание 4. Если входные данные исходной системы (1)-(5) удовлетворяют условиям теоремы 1 (в частности, $a=a(x, t), e(t, u)=0, h(t, u)=0)$, то требования леммы 5 к функциям $\gamma(x, t, u)$ и $\rho^{0}(x)$ позволяют утверждать, что дифференциально-разностная система (8)-(12) имеет единственное решение $\left\{u_{n}(x), \rho_{n}(x)\right\}$ в более гладком классе функций, а именно, $u_{n}(x)$ принадлежит $H_{\tau}^{2+\lambda, 1+\lambda / 2}$ во всей замкнутой области $\bar{Q}_{\tau},|\hat{u}(x)|_{Q_{\tau}}^{2+\lambda, 1+\lambda / 2} \leqslant M_{4}, M_{4}>0$-постоянная из оценки (41).

4.2. Полученные результаты для решения $\left\{u_{n}(x), \rho_{n}(x)\right\}$ дифференциально-разностной системы (8)-(12) позволяют сформулировать условия существования и единственности решения $\{u(x, t), \rho(x, t)\}$ нелинейной параболической системы (1)-(5).

В случае $a=a(x, t), e(t, u)=0, h(t, u)=0$ имеет место

Теорема 3. Предположим, что:

1) при $(x, t) \in \bar{Q}$ и любых $u,|u|<\infty$, все входные данные в соотношениях (1)-(4) являются ограниченными функииями в областях своего определения, причем коэффициент $a(x, t)$ ограничен в $\bar{Q}$ вместе со своими производными по $x$ u $t$, выполнены условия $0<a_{\min } \leqslant a \leqslant a_{\max }, 0<c_{\min } \leqslant c \leqslant c_{\max }, h=0$, $e=0$; кроме того, функиии $a_{x}(x, t)$ u $f(x, t)$ принадлежат $H^{\lambda, \lambda / 2}(\bar{Q})$;

2) при $(x, t, u) \in \bar{D}=Q \times\left[-M_{0}, M_{0}\right]\left(M_{0}>0-\right.$ постоянная из оченки (13)) функции $b(x, t, u) u$ $d(x, t, u)$ непрерывны в смысле Гельдера по $x$ и $t$ с показателями $\lambda, \lambda / 2$ и имеют ограниченные производные по и; кроме того, коэфбичиент $c(x, t, u)$ принадлежит $H^{1, \lambda / 2,1}(\bar{D})$;

3) функиии $\gamma(x, t, u)$ и $\rho^{0}(x)$ в условии (5) принадлежат пространствам $H^{1, \lambda / 2,1}(\bar{D})$ u $C^{1}[0, l]$ соответственно, $0<\rho_{\min }^{0} \leqslant \rho^{0}(x) \leqslant \rho_{\max }^{0}, \rho_{\min }^{0}, \rho_{\max }^{0}=$ const $>0$;

4) бункиии $\varphi(x), g(t)$ и $q(t)$ принадлежат соответственно пространствам $H^{2+\lambda}[0, l]$ u $O^{1}[0, T] u$ удовлетворяют условиям согласования при $t=0:\left.a(x, 0) \varphi_{x}\right|_{x=0}=g(0),\left.a(x, 0) \varphi_{x}\right|_{x=l}=q(0)$.

Тогда нелинейная система (1)-(5) имеет единственное гладкое решение $\{u(x, t), \rho(x, t)\}$, обладающее свойствами

$$
\begin{gathered}
u(x, t) \in H^{2+\lambda, 1+\lambda / 2}(\bar{Q}), \quad|u(x, t)|_{\bar{Q}}^{2+\lambda, 1+\lambda / 2} \leqslant M_{4}, \quad M_{4}=\text { const }>0 \quad \text { из оченки }(41), \\
\rho(x, t) \in C(\bar{Q}), \quad \rho_{x}(x, t) \in C(\bar{Q}), \quad \rho_{t}(x, t) \in H^{\lambda, \lambda / 2}(\bar{Q})
\end{gathered}
$$

и удовлетворяющее ограничениям (6), (7) в зависимости от знака функции $\gamma(x, t, u)$, и его можно получить как предел решения $\left\{u_{n}(x), \rho_{n}(x)\right\}$ дифберенциально-разностной системъ (8)-(12) при стремлении шага сетки $\tau$ к нулю по некоторой подпоследовательности шагов $\tau_{j}$.

Условия однозначной разрешимости системы (1)-(5) для общего случая $a=a(x, t, u), h(t, u)>0$, $e(t, u)>0$ устанавливает

Теорема 4. Предположим, что:

1) при $(x, t) \in \bar{Q}$ и любых $u,|u|<\infty$, все входные данные в соотношениях (1)-(4) являются ограниченными функииями в областях своего определения, причем коэффициент $a(x, t, u)$ ограничен вместе со своими производными по $x$ и $u$, выполнены условия $0<a_{\min } \leqslant a \leqslant a_{\max }, 0<c_{\min } \leqslant c \leqslant c_{\max }, h(t, u) \geqslant 0$, $e(t, u) \geqslant 0$; кроме того, функция $f(x, t)$ имеет ограниченную производную по $x$ и непрерывна в смысле Гельдера по $t$ с показателем $\lambda / 2$;

2) при $(x, t, u) \in \bar{D}=Q \times\left[-M_{0}, M_{0}\right]\left(M_{0}>0-\right.$ постоянная из оченки (13)) функиии $a(x, t, u)$, $a_{x}(x, t, u), a_{u}(x, t, u), b(x, t, u), c(x, t, u) u d(x, t, u)$ принадлежат $H^{1, \lambda / 2,1}(\bar{D}) ; \oint у н к u и и(t, u) u e(t, u)$ имеют ограниченные производные по $t u u$;

3) функиии $\gamma(x, t, u)$ и $\rho^{0}(x)$ в условии (5) принадлежат пространствам $H^{1, \lambda / 2,1}(\bar{D})$ u $C^{1}[0, l]$ соответственно, $0<\rho_{\min }^{0} \leqslant \rho^{0}(x) \leqslant \rho_{\max }^{0}, \rho_{\min }^{0}, \rho_{\max }^{0}=$ const $>0$;

4) бункиии $\varphi(x), g(t)$ и $q(t)$ принадлежат соответственно пространствам $H^{2+\lambda}[0, l] u O^{1}[0, T] u$ удовлетворяют условиям согласования при $t=0$ :

$$
\left.a(x, 0, \varphi) \varphi_{x}\right|_{x=0}-\left.h(0, \varphi) \varphi\right|_{x=0}=g(0),\left.\quad a(x, 0, \varphi) \varphi_{x}\right|_{x=l}+\left.e(0, \varphi) \varphi_{x}\right|_{x=l}=q(0)
$$

Тогда нелинейная система (1)-(5) имеет хотя бы одно гладкое решение $\{u(x, t), \rho(x, t)\}$, обладающее 
свойствами

$$
\begin{gathered}
u(x, t) \in C(\bar{Q}), \quad u_{x}(x, t) \in C(\bar{Q}), \\
u(x, t) \in H^{2+\lambda, 1+\lambda / 2}(Q) \quad n p u \quad 0<x<l, \quad 0 \leqslant t \leqslant T, \\
|u(x, t)|_{Q}^{2+\lambda, 1+\lambda / 2} \leqslant M_{5}, \quad M_{5}=\text { const }>0 \quad \text { из оценки } \quad(42), \\
\rho(x, t) \in C(\bar{Q}), \quad \rho_{x}(x, t) \in C(\bar{Q}), \quad \rho_{t}(x, t) \in H^{\lambda, \lambda / 2}(\bar{Q}),
\end{gathered}
$$

и удовлетворяющее ограничениям (6), (7) в зависимости от знака функции $\gamma(x, t, u)$, и его можно получить как предел решения $\left\{u_{n}(x), \rho_{n}(x)\right\}$ дифференциально-разностной системь (8)-(12) при стремлении шага сетки $\tau$ к нулю по некоторой подпоследовательности шагов $\tau_{j}$.

При выполнении входными данными условий (43) решение $\{u(x, t), \rho(x, t)\}$ является единственным в этом классе функций.

Ограничимся изложением доказательства этой теоремы, так как обоснование теоремы 3 проводится аналогично, только с соответствующими упрощениями.

Доказательство теоремы 4. Прежде всего заметим, что равномерные оценки (42), (49) и (50) означают компактность семейства $\left\{u_{n}(x), \rho_{n}(x)\right\}$ в соответствующих пространствах. Это позволяет, проводя обычные рассуждения при совершении предельного перехода при $\tau_{j} \rightarrow 0$ (т.е. при $n_{j} \rightarrow \infty$ ) в условиях (8)-(12), установить существование решения $\{u(x, t), \rho(x, t)\}$ системы $(1)-(5)$, такого, что $u(x, t) \in$ $H^{2+\lambda, 1+\lambda / 2}(Q)$ при $0<x<l, 0 \leqslant t \leqslant T, \rho(x, t) \in C(\bar{Q}), \rho_{t}(x, t) \in H^{\lambda, \lambda / 2}(\bar{Q})$. Кроме того, оценки (49) позволяют установить, что $\rho(x, t)$ удовлетворяет неравенствам (6) и $(7)$ в зависимости от знака функции $\gamma(x, t, u)$ в условии $(5)$.

Покажем, что предположения теоремы 4 о входных данных позволяют утверждать, что $u(x, t)$ и $\rho(x, t)$ обладают большей гладкостью. Так, производная $u_{x}(x, t)$ непрерывна во всей замкнутой области $\bar{Q}_{\tau}$, т.е. $\lim _{x \rightarrow 0} u_{x}(x, t)=u_{x}(0, t), \lim _{x \rightarrow l} u_{x}(x, t)=u_{x}(l, t)$. Доказательство этого утверждения с учетом оценок $\max _{(x, t) \in \bar{Q}}|u(x, t)| \leqslant M_{0}, \max _{(x, t) \in \bar{Q}}\left|u_{x}(x, t)\right| \leqslant M_{1}$ основано на методе барьеров и повторяет аналогичные рассуждения, проведенные в теореме 2. Требования же гладкости функций $\gamma(x, t, u)$ и $\rho^{0}(x)$ в условии (5) позволяют доказать, что решение $\rho(x, t)$ обладает непрерывной в $\bar{Q}$ производной $\rho_{x}(x, t)$. Действительно, из (5) следует, что при $0 \leqslant t \leqslant T$

$$
\begin{gathered}
\rho(x, t)=\int_{0}^{t} \gamma(x, \tau, u(x, \tau)) d \tau+\rho^{0}(x), \\
\rho_{x}(x, t)=\int_{0}^{t}\left\{\gamma_{x}(x, \tau, u(x, \tau))+\gamma_{u}(x, \tau, u(x, \tau)) u_{x}(x, \tau)\right\} d \tau+\rho_{x}^{0}(x) .
\end{gathered}
$$

Таким образом, непрерывность $\rho_{x}(x, t)$ в $\bar{Q}$ является следствием непрерывности $u_{x}(x, t)$ в $\bar{Q}$ и принадлежности функций $\gamma(x, t, u)$ и $\rho^{0}(x)$ классам $H^{1, \lambda / 2,1}(\bar{D})$ и $C^{1}[0, l]$ соответственно.

Итак, доказано существование решения $\{u(x, t), \rho(x, t)\}$ системы $(1)-(5)$, обладающего свойствами гладкости, указанными в теореме 4.

Покажем теперь, что решение $\{u(x, t), \rho(x, t)\}$ единственно в классе гладких функций, таких, что

$$
\sup _{(x, t) \in \bar{Q}}\left|u, u_{x}, u_{x x}, u_{t}\right|<\infty, \quad \sup _{(x, t) \in \bar{Q}}\left|\rho, \rho_{x}, \rho_{t}\right|<\infty
$$

Предположим, что при $t \in\left[0, t^{0}\right], 0 \leqslant t^{0}<T$, единственность решения $\{u(x, t), \rho(x, t)\}$ уже доказана. Покажем, что тогда единственность имеет место и для $t \in\left[t^{0}, t^{0}+\Delta t\right]$, где $\Delta t>0-$ достаточно малая, но фиксированная величина, что позволяет за конечное число шагов исчерпать весь отрезок $[0, T]$. Допустим противное, т.е. что при $t \in\left[t^{0}, t^{0}+\Delta t\right]$ существуют два решения системы (1)- $(5):\{u(x, t), \rho(x, t)\}$ и $\{\bar{u}(x, t), \bar{\rho}(x, t)\}$. Выражения для $\rho(x, t)$ и $\bar{\rho}(x, t)$ при $t \in\left[t^{0}, t^{0}+\Delta t\right]$ имеют вид (см. $\left.(52)\right)$

$$
\rho(x, t)=\int_{t^{0}}^{t^{0}+\Delta t} \gamma(x, \tau, u(x, \tau)) d \tau+\rho\left(x, t^{0}\right), \quad \bar{\rho}(x, t)=\int_{t^{0}}^{t^{0}+\Delta t} \gamma(x, \tau, \bar{u}(x, \tau)) d \tau+\bar{\rho}\left(x, t^{0}\right) .
$$


Так как по предположению $\rho\left(x, t^{0}\right)=\bar{\rho}\left(x, t^{0}\right)$, то для разностей

$$
v(x, t)=u(x, t)-\bar{u}(x, t), \quad \zeta(x, t)=\rho(x, t)-\bar{\rho}(x, t)
$$

в области $\bar{Q}_{t^{0}}=\left\{0 \leqslant x \leqslant l, t^{0} \leqslant t \leqslant t^{0}+\Delta t\right\}$ следует оценка

$$
\max _{(x, t) \in \bar{Q}_{t^{0}}}|\zeta(x, t)| \leqslant \Delta t \max _{(x, t, u) \in \bar{D}}\left|\gamma_{u}(x, t, u)\right| \max _{(x, t) \in \bar{Q}_{t^{0}}}|v(x, t)| .
$$

Кроме того, для $v(x, t)$ и $\zeta(x, t)$ в силу $(1)-(4)$ имеют место соотношения

$$
\begin{gathered}
c(x, t, u) \rho(x, t) v_{t}-\left(a(x, t, u) v_{x}\right)_{x}+\mathcal{A}_{0} v_{x}+\mathcal{A}_{1} v=c(x, t, \bar{u}) \bar{u}_{t} \zeta(x, t), \quad(x, t) \in Q_{t^{0}}, \\
a(x, t, u) v_{x}-\left.\mathcal{A}_{2} v\right|_{x=0}=0, \quad t^{0}<t \leqslant t^{0}+\Delta t, \\
a(x, t, u) v_{x}+\left.\mathcal{A}_{3} v\right|_{x=l}=0, \quad t^{0}<t \leqslant t^{0}+\Delta t, \\
v\left(x, t^{0}\right)=0, \quad 0 \leqslant x \leqslant l,
\end{gathered}
$$

в которых коэффициенты $\mathcal{A}_{0}$ и $\mathcal{A}_{1}$ зависят соответствующим образом от производных $a_{u}, a_{x u}, a_{u u}, b_{u}, c_{u}$ и $d_{u}$ в точке $(x, t, \sigma u+(1-\sigma) \bar{u})(0<\sigma<1)$, а также от $\rho(x, t), u(x, t)$ и производных $u_{x}(x, t), u_{x x}(x, t)$ и $u_{t}(x, t)$. Коэффициенты $\mathcal{A}_{2}$ и $\mathcal{A}_{3}$ в граничных условиях зависят соответствующим образом от производных по $u$ функций $a(x, t, u), h(t, u)$ и $e(t, u)$, а также от $u(x, t)$ и $u_{x}(x, t)$ при $x=0$ и $x=l$.

Все входные данные этой линейной краевой задачи равномерно ограничены в области $\bar{Q}_{t^{0}}$ как функции $(x, t)$, причем выполнение ими условия (43) приводит к неотрицательности коэффициентов $\mathcal{A}_{2} \geqslant 0$, $\mathcal{A}_{3} \geqslant 0$. Это позволяет применить принцип максимума и получить оценку

$$
\max _{(x, t) \in \bar{Q}_{t^{0}}}|v(x, t)| \leqslant \mathcal{K}_{0} \max _{(x, t) \in \bar{Q}_{t^{0}}}|\zeta(x, t)|, \quad \mathcal{K}_{0}=\text { const }>0 .
$$

Отсюда и из (53) следует, что

$$
\max _{(x, t) \in \bar{Q}_{t^{0}}}|v(x, t)| \leqslant \Delta t \mathcal{K}_{0} \max _{(x, t, u) \in \bar{D}}\left|\gamma_{u}(x, t, u)\right| \max _{(x, t) \in \bar{Q}_{t^{0}}}|v(x, t)| .
$$

Выбирая затем величину $\Delta t>0$ из условия $\Delta t \mathcal{K}_{0} \underset{(x, t, u) \in \bar{D}}{\max }\left|\gamma_{u}(x, t, u)\right| \leqslant 1-\mu, 0<\mu<1$, получим из (54) соотношение

$$
\max _{(x, t) \in \bar{Q}_{t^{0}}}|v(x, t)| \leqslant(1-\mu) \max _{(x, t) \in \bar{Q}_{t^{0}}}|v(x, t)|,
$$

т.е. $\max _{(x, t) \in \bar{Q}_{t^{0}}}|v(x, t)|=0$. Но это означает в силу (53), что и $\max _{(x, t) \in \bar{Q}_{t^{0}}}|\zeta(x, t)|=0$.

Таким образом, предположение о неединственности решения системы (1)-(5) при $t \in\left[t^{0}, t^{0}+\Delta t\right]$ приводит к противоречию.

Повторяя подобные рассуждения для отрезков $t \in\left[t^{1}, t^{2}\right]\left(t^{1}=t^{0}+\Delta t, t^{2}=t^{1}+\Delta t\right), t \in\left[t^{2}, t^{3}\right]$ и т.д. вПлоть до конечного момента времени $T$, установим единственность решения системы (1)-(5) на всем отрезке $[0, T]$. Теорема 4 доказана.

4.3. Завершая обоснование метода прямых Ротэ как способа решения нелинейной параболической системы (1)-(5), оценим его погрешность, т.е. получим оценки для разностей

$$
w_{n}(x)=u_{n}(x)-u\left(x, t_{n}\right), \quad \xi_{n}(x)=\rho_{n}(x)-\rho\left(x, t_{n}\right)
$$

$\left\{u\left(x, t_{n}\right), \rho\left(x, t_{n}\right)\right\}$ - точное решение исходной системы $(1)-(5)$ в момент времени $t=t_{n},\left\{u_{n}(x), \rho_{n}(x)\right\}-$ точное решение ее дифференциально-разностного аналога (8)-(12). Имеет место

Теорема 5. Пусть входные данные удовлетворяют условиям теоремы 4. Тогда для погрешности метода прямых Ротэ при любом достаточно малом шаге сетки $\tau$ справедлива оченка

$$
\max _{\left(x, t_{n}\right) \in \bar{Q}_{\tau}}\left|w_{n}(x)\right| \leqslant \mathcal{K}_{1}(\Psi+\psi), \quad \max _{\left(x, t_{n}\right) \in \bar{Q}_{\tau}}\left|\xi_{n}(x)\right| \leqslant \mathcal{K}_{2}(\Psi+\psi)
$$

где $\Psi=\max _{\left(x, t_{n}\right) \in \bar{Q}_{\tau}} \Psi_{n}(x), \psi=\max _{\left(x, t_{n}\right) \in \bar{Q}_{\tau}} \psi_{n}(x), \Psi_{n}(x)$ - погрешность аппроксимации дифберенциальноразностной краевой задачи (8)-(11), $\psi_{n}(x)$ - погрешность аппроксимации уравнения $(12), \mathcal{K}_{1}>0 u$ $\mathcal{K}_{2}>0$ - постоянные, не зависящие от $x, t, \tau, n$. 
Доказательство теоремы 5 опускаем. Оно повторяет с соответствующими модификациями доказательство единственности, приведенное выше. Отметим только, что оценки (55) устанавливаются последовательно для конечных отрезков времени $\left[0, t_{n_{0}}\right],\left[t_{n_{0}}, t_{n_{1}}\right],\left[t_{n_{1}}, t_{n_{2}}\right]$ и т.д. вплоть до момента $t_{N}=T$. Наличие таких оценок позволяет рассматривать изложенную схему метода Ротэ как конструктивный способ приближенного решения нелинейной параболической системы (1)-(5).

5. Математическая модель физико-химического процесса термодеструкции. Различные постановки системы (1)-(5) возникают при моделировании физико-химических процессов, связанных с изменениями внутренних характеристик материалов, в которых эти процессы протекают. В качестве одного из приложений такой математической модели можно привести изменение фильтрационных свойств пластов при современных способах разработки нефтегазовых месторождений. Остановимся на других приложениях, связанных с использованием композиционных материалов в системах теплозащиты технических объектов, подвергающихся воздействию высоких температур (аэрокосмические аппараты, энергоустановки и т.п.).

При высокотемпературном нагреве теплозащитный композиционный материал испытывает разнообразные физико-химические превращения и подвергается деструкции - необратимым изменениям внутренних параметров (таких как плотность, концентрация компонентов композита и т.п.).

Приведем математическую модель процесса разложения термодеструктирующего материала пластины конечной толщины под воздействием теплового потока. В основе модели - представление этого процесса с помощью аппроксимации суммой нескольких стадий (реакций) с различными кинетическими параметрами $[6,7]$. Соответствующая задача термодеструкции состоит в нахождении температуры и концентрации компонентов композита $\{u(x, t), \rho(x, t)\}$ из условий:

$$
\begin{gathered}
c(u) \rho(x, t) u_{t}(x, t)-\left(\lambda(u) u_{x}\right)_{x}=0, \quad(x, t) \in Q=\{0<x<l, 0<t \leqslant T\}, \\
\left.\lambda(u) u_{x}\right|_{x=0}=0, \quad \lambda(u) u_{x}+\left.\epsilon \sigma u^{4}\right|_{x=l}=q(t), \quad 0<t \leqslant T, \\
\left.u\right|_{t=0}=\varphi(x), \quad 0 \leqslant x \leqslant l, \\
\rho(x, t)=\sum_{s=1}^{S} \rho^{s}(x, t), \quad \rho^{s}(x, t)=\rho_{0}^{s}(x) \exp \left(-A^{s} \int_{0}^{t} \exp \left(-\frac{E^{s}}{u(x, \tau)}\right) d \tau\right),
\end{gathered}
$$

в которых $c(u)$ и $\lambda(u)$ - теплофизические характеристики пластины (коэффициенты теплоемкости и теплопроводности), $q(t)$ - плотность теплового потока, $\sigma$ - константа Стефана-Больцмана, $\epsilon$ - степень черноты, $S$ - число стадий (реакций) композита, $A^{s}$ и $E^{s}-$ кинетические параметры $s$-ой стадии, $\rho^{s}(x, t)-$ концентрация $s$-ой стадии, $\varphi(x)>0$ и $\rho_{0}^{s}(x)>0$ - начальные распределения температуры и концентраций стадий, $l$ - толщина пластины, $T$ - время теплового воздействия.

Математические модели, описывающие процесс разложения других параметров композиционного материала, отличаются видом условия (59). Например, если $\rho(x, t)-$ распределение плотности этого материала, то условие (59) принимает вид

$$
\rho(x, t)=\rho_{0}(x) \exp \left(-A \int_{0}^{t} \exp \left(-\frac{E}{u(x, \tau)}\right) d \tau\right), \quad \rho_{0}(x)>0,
$$

где $\rho_{0}(x)$ - начальная плотность композиционного материала (до теплового воздействия).

Применительно к аэрокосмической технике все эти модели связаны с исследованием состояния системы теплозащиты спускаемого аппарата. Возвращаясь к задаче (56)-(59), представим наши результаты численного моделирования процесса деструкции образца из композиционного материала (число стадий $S=5)$ с заданными теплофизическими и кинетическими характеристиками. На поверхность образца толщиной $l$, равной $3.5 \times 10^{-3}$ метров, в течение времени $T$, равного 20 секундам, воздействует тепловой поток

$$
q(t)= \begin{cases}1.8 t, & 0 \leqslant t \leqslant 5 \\ 1.8(10-t), & 5 \leqslant t \leqslant 10 \\ 3(t-10), & 10 \leqslant t \leqslant 15 \\ 3(20-t), & 15 \leqslant t \leqslant 20\end{cases}
$$

За время этого теплового воздействия происходит изменение температурного режима в образце с $300^{0}$ в начальный момент $t=0$ до почти $600^{0}$ в конечный момент $t=T$. Нагрев сопровождается разрушением 
компонентов композита (см. таблицу). Особенно существенной деструкции подверглись две стадии $(s=2$ и $s=3)$.

Концентрации стадий на поверхности образца в моменты времени $t=0$ и $t=T$

\begin{tabular}{||c|c|c|c|c|c||}
\hline \hline$s$-я стадия & $\rho^{1}(l, t)$ & $\rho^{2}(l, t)$ & $\rho^{3}(l, t)$ & $\rho^{4}(l, t)$ & $\rho^{5}(l, t)$ \\
\hline При $t=0$ & $3.811 \times 10^{-2}$ & $6.302 \times 10^{-1}$ & $2.561 \times 10^{-1}$ & $4.132 \times 10^{-2}$ & $2.124 \times 10^{-2}$ \\
\hline При $t=T$ & $3.552 \times 10^{-2}$ & $3.635 \times 10^{-2}$ & 0. & $3.762 \times 10^{-3}$ & $4.721 \times 10^{-3}$ \\
\hline \hline
\end{tabular}

6. Заключение. Исследована нелинейная параболическая система, в которой в отличие от обычных постановок краевых задач требуется определить еще и неизвестный коэффициент при производной по времени в параболическом уравнении. Полученные результаты позволяют обосновать математическую постановку этой системы и указать условия ее однозначной разрешимости в классе гладких функций. Эти условия связаны с применением метода прямых Ротэ и с соответствующими априорными оценками для решений нелинейных дифференциально-разностных краевых задач в сеточно-непрерывных классах Гельдера. Для одного из видов входных данных предложен способ получения оценки производной решения такой краевой задачи, учитывающий свойства входных данных и позволяющий ослабить требования гладкости от этих данных.

Указаны области практического применения рассмотренной параболической системы и приведены конкретные примеры, связанные с использованием композиционных материалов в современных системах теплозащиты.

\title{
СПИСОК ЛИТЕРАТУРЫ
}

1. Ладыюенская О.А., Солонников В.А., Уральцева Н.Н. Линейные и квазилинейные уравнения параболического типа. М.: Наука, 1967.

2. Gol'dman N.L. Inverse Stefan problems. Dordrecht: Kluwer Academic Publishers, 1997.

3. Гольдман Н.Л. Обратные задачи Стефана. Теория и методы решения. М.: Изд-во Моск. ун-та, 1999.

4. Кружков C.Н. Априорная оценка для производной решения параболического уравнения // Вестник Моск. ун-та. Серия 1: Математика. Механика. 1967. № 2. 41-48.

5. Канторович Л.В., Акилов Г.П. Функциональный анализ. М.: Наука, 1984.

6. Алексеев A.K. О восстановлении истории нагрева пластины из термодеструктирующего материала по профилю плотности в конечном состоянии // Теплофизика высоких температур. 1993. 31, № 6. 975-979.

7. Alekseev A.K. Heat memory of structures with phase transitions // J. Intelligent Material Systems and Structures. 1994. 5, N 1. 90-94.

Поступила в редакцию 21.06.2017

\section{A Nonlinear Problem for a Parabolic Equation with an Unknown Coefficient at the Time Derivative and its Applications in Mathematical Models of Physico-Chemical Processes}

\author{
N. L. Gol'dman ${ }^{1}$ \\ ${ }^{1}$ Lomonosov Moscow State University, Research Computing Center; Leninskie Gory, Moscow, 119992, \\ Russia;Dr. Sci., Leading Scientist,e-mail: goldman@srcc.msu.ru
}

Received June 21, 2017

Abstract: We consider conditions of unique solvability in a class of smooth functions for a nonlinear system with an unknown coefficient at the time derivative in a parabolic equation. To this end, the Rothe method is applied, which provides not only the proof of solvability but also the constructive solution of the considered system. A priori estimates in the grid-continuous Hölder spaces are established for the corresponding differentialdifference nonlinear system that approximates the initial parabolic system by the Rothe method. Such estimates allow one to prove the existence of the smooth solution of this parabolic system and to obtain the error estimates 
for the Rothe method. This study is connected with the mathematical modelling of physico-chemical processes where the inner characteristics of materials are subjected to changes. As an example, the problem on the destruction of a heat-protective composite under the effect of high-temperature heating is discussed.

Keywords: parabolic equations, Hölder spaces, Rothe method, a priori estimates, unique solvability, mathematical model, thermodestruction, composite.

\section{References}

1. O. A. Ladyzhenskaya, V. A. Solonnikov, and N. N. Ural'tseva, Linear and Quasilinear Equations of Parabolic Type (Nauka, Moscow, 1967; SIAM, Providence, 1968).

2. N. L. Gol'dman, Inverse Stefan Problems (Kluwer, Dordrecht, 1997).

3. N. L. Gol'dman, Inverse Stefan Problems. Theory and Methods of Solution (Mosk. Gos. Univ., Moscow, 1999) [in Russian].

4. S. N. Kruzhkov, "A Priori Estimate for the Derivative of a Solution to a Parabolic Equation," Vestn. Mosk. Univ., Ser. 1: Mat. Mekh., No. 2, 41-48 (1967).

5. L. V. Kantorovich and G. P. Akilov, Functional Analysis (Nauka, Moscow, 1977; Pergamon, New York, 1982).

6. A. K. Alekseev, "On the Restoration of the Heating History of a Plate Made of a Thermodestructible Material from the Density Profile in the Final State," Teplofiz. Vys. Temp. 31 (6), 975-979 (1993) [High Temp. 31 (6), 897-901 (1993)].

7. A. K. Alekseev, "Heat Memory of Structures with Phase Transitions," J. Intell. Mater. Syst. Struct. 5 (1), 90-94 (1994). 